\title{
5. Freiwilliges und zivilgesellschaftliches Engagement - Stand der Forschung und aktuelle empirische Befunde
}

Die Begriffe Zivilgesellschaft und das damit verbundene freiwillige resp. zivilgesellschaftliche Engagement sind seit vielen Jahren Gegenstand zahlreicher Diskussionen, Projekte und Konzepte. ,Sowohl in den öffentlichen Debatten als auch in der sozialwissenschaftlichen Forschung"(Olk/Hartnuss 2011: 145) haben diese Begriffe seit Mitte der 1980er Jahre an Bedeutung gewonnen.

In der Fachdiskussion haben sich hierfür verschiedene theoretische Orientierungen und Konzepte mit jeweils unterschiedlichen Bedeutungen etabliert. So wird der Begriff Zivilgesellschaft in den Politik- und Sozialwissenschaften normativ und analytisch verwendet. Er verweist dann auf die Rolle der Zivilgesellschaft beim Übergang von autoritären Regimen zu Demokratien, oder fragt nach der Bedeutung einer unabhängigen Zivilgesellschaft für eine lebendige und starke Demokratie. (Adloff 2005: 7) Politikerinnen und Politiker und Bürgerrechtlerinnen und Bürgerrechtler hingegen gebrauchen „Zivilgesellschaft“ als ,politischen Signalbegriff“. Auf der einen Seite steht er für ein demokratisches Regulativ gegen einen starken Staat, auf der anderen Seite für die Vorstellung, dass die Bürgerinnen und Bürger sich nicht allein auf den Sozialstaat verlassen, sondern ihre Angelegenheiten eigenverantwortlich in die Hand nehmen sollten. (ebd: 7) Insofern erscheint eine lebendige Zivilgesellschaft als Garant dafür, dass die gesellschaftlichen Gruppen nicht zu weit auseinanderdriften. (Enquete-Kommission „Zukunft des bürgerschaftlichen Engagements“ 2002: 40)

Im folgenden Kapitel werde ich näher auf die verschiedenen Begriffe von Zivilgesellschaft und die damit verbundenen Konzepte des zivilgesellschaftlichen bzw. freiwilligen Engagements eingehen. Ich beziehe mich im Folgenden ${ }^{11}$ deshalb auf die Verknüpfung und wechselseitige Durchdringung von Zivilgesellschaft und Engagement.

\subsection{Idee und Konzept der Zivilgesellschaft}

\section{Unterschiedliche und sich wandelnde Auffassungen von Zivilgesellschaft}

Der Begriff Zivilgesellschaft oder bürgerliche Gesellschaft, der auf Aristoteles’ Begriff der politiké koinonia (lat. societas civilis) zurückgeht, bedeutet wörtlich übersetzt so viel wie Bürgervereinigung oder Bürgergemeinde. Nach der antiken Auffassung steht ,Zivilgesellschaft“ somit für ,eine Gemeinschaft von Bürgern, die sich zum Zweck des 'guten', in diesem Fall des tugendhaften und glücklichen Lebens zusammenschliessen“. (Adloff 2005: 17) Die Zivilgesellschaft wird hier synonym mit dem Herrschaftsverband der athenischen Bürgergemeinde (Polis) verwendet, in dem tugendhafte, männliche und freie Bürger zusammenkommen, um sich für

\footnotetext{
${ }^{11}$ Die folgenden Kapitel orientieren sich an früheren Überlegungen, die ich zusammen mit Sonja Kubisch in unserer Monografie „Erfahrungswissen in der Zivilgesellschaft“ bereits publiziert habe. (vgl. Kubisch/Störkle 2016: 33ff.)
}

(C) Der/die Autor(en) 2021

M. Störkle, Expatriates und freiwilliges Engagement in der

Schweiz, https://doi.org/10.1007/978-3-658-33043-9_5 
öffentliche Belange einzusetzen. (ebd.: 18) Trotz der Bedeutungsverschiebungen und -erweiterungen wird der Begriff Zivilgesellschaft weiterhin als Bezeichnung für politische Selbstorganisation und demokratische Partizipation genutzt. (Adloff 2005: 18)

Alexis de Tocqueville, ein weiterer Klassiker der politischen Philosophie, ist für die Theoriegeschichte der Zivilgesellschaft hoch bedeutsam. Er setzt sich in seinem Werk „Über die Demokratie in Amerika“ (1985/1835) mit der Bedeutung von Vereinigungen (er spricht von „Assoziationen") für das amerikanische Gemeinwesen auseinander. Es sind diese freien Zusammenschlüsse, d. h. Assoziationen oder Vereine, die, wie Tocqueville bemerkt, die Demokratie mit Leben erfüllen und erhalten. (Adloff 2005: 39) Fasziniert beobachtet er, dass sich in den Vereinigten Staaten die Menschen zusammenschliessen, um gemeinsam politische oder soziale Ziele zu verfolgen, um auf lokaler Ebene auf die Politik Einfluss zu nehmen oder um das zu behandelnde Problem selbst zu lösen. Für Tocqueville haben auch Vereinigungen einen hohen demokratischen Wert, die keinen politischen, sondern ,nur“ geselligen Zwecken dienen. Nach seiner festen Überzeugung fördern diese Vereinigungen die Tugend und die „Demokratiefähigkeit“ der Bürger*innen, wobei das „Miteinander-Handeln“ essenziell ist. (ebd.: 40) Rein gesellige Vereinigungen haben deswegen eine hohe politische Bedeutung. Da die Menschen in den Vereinigten Staaten, wie Tocqueville feststellt, von den feudalen Fesseln ständischer Ordnung emanzipiert sind und vorwiegend zu individualistischen und egalitaristischen Orientierungen neigen, sind sie gezwungen, sich zusammenzuschliessen und zueinander neue Bande zu entwickeln, um dem drohenden demokratischen Despotismus zu entrinnen. Diese „Kunst der Vereinigung“ hilft dann so etwas wie Bürgertugend und Gemeinsinn zu vermitteln. Diese Vereinigungen fasst Tocqueville als eine intermediäre Ebene zwischen vereinzelten Individuen und dem Staat auf, sie ,stellen mithin den entscheidenden Unterbau einer Demokratie dar und konstituieren die Zivilgesellschaft“. (ebd.: 40) Somit weist Tocqueville der Zivilgesellschaft vor allem subsidiäre Funktionen gesellschaftlicher und politischer Selbstorganisation zu. (ebd.: 40) Dieses liberale Verständnis von Zivilgesellschaft setzt sich allerdings erst zwischen 1750 und 1850 mit den Autoren der Aufklärung wie Locke, Montesquieu und Ferguson durch. Wie Jürgen Kocka scharfsinnig hervorhebt, bezeichnet Zivilgesellschaft zu dieser Zeit einen Entwurf des friedlichen, zivilisierten Zusammenlebens freier Bürger*innen ,ohne obrigkeitsstaatliche Gängelung“. (Kocka 2003: 30, zit. in Adloff 2005: 9)

Als Karl Marx den Begriff Zivilgesellschaft auf die bürgerliche Gesellschaft der Eigentümer*innen von Produktionsmitteln reduzierte und ihm damit einen neuen Bedeutungsgehalt zuwies, geriet das Konzept nach Adloff zunehmend an den Rand des Interesses. (2005: 9) Anfang des 20. Jahrhunderts erlebt der Begriff Zivilgesellschaft eine Renaissance - aufgrund der Weiterentwicklung durch Antonio Gramsci und John Dewey. Der amerikanische pragmatische Philosoph Dewey ist der Meinung, Demokratie zeichne sich durch eine starke Zivilgesellschaft aus. Bei beiden ist bereits eine Abgrenzung gegenüber der Wirtschaft impliziert, denn sie betonen in ihren Texten immer wieder die Bedeutung von freien nicht-staatlichen und nicht-kapitalistischen Assoziationen. (ebd.: 9)

Der Begriff bürgerliche Gesellschaft stand in Deutschland lange Zeit in der Tradition von Hegel und Marx, die in ihren Theorien den „Primat der Ökonomie“ postulierten. Im Unterschied dazu 
geht das moderne Verständnis von drei getrennten Sphären - „Wirtschaft“, „Staat“ und „Zivilgesellschaft“ - aus. (Klein 2011: 32) Der Begriff „civil society“ hat in der angelsächsischen Welt eine andere theoretische Ausrichtung. Da Kontinentaleuropa historisch mit der Tradition eines starken Staates konfrontiert war, bezog sich hier das Konzept „bürgerliche Gesellschaft“ immer auch auf die Unterscheidung von „Staat“ und „Gesellschaft“. Da der angelsächsische Kulturraum einen anderen theoretischen Bezugspunkt setzte, findet sich in den Konzeptionen der „civil society“ seit John Locke schon ,das Bemühen in ganz eigenständiger Weise die zivilisierende Kraft gesellschaftlicher Entwicklungen und auch von Akteurszusammenhängen zu beschreiben und deren Folgen für Wirtschaft und Staat zu analysieren“. (ebd.: 32) Die neuere Diskussion über die Zivilgesellschaft in unserem Sprachraum ist vor allem gekennzeichnet durch die in der deutschen Sprache mögliche Differenzierung von ,bürgerlicher“ und ,ziviler Gesellschaft“. Das wesentliche Motiv für die Bevorzugung des Neologismus der „Zivilgesellschaft“ liegt, so Ansgar Klein, im Bestreben, sich vom Begriff der „,bürgerlichen Gesellschaft“" abzugrenzen. (ebd.: 32)

\section{Gegenwärtige Diskurse über Zivilgesellschaft}

Der neuere Diskurs zur Zivilgesellschaft entwickelt sich in den späten 1970er Jahren. (Klein 2001: 19ff.) Ansgar Klein (2011) charakterisiert diesen Diskurs als ,eine weit ausholende und unabgeschlossene theoretische Suchbewegung nach den politischen Handlungsmöglichkeiten gesellschaftlicher Akteure zur Herstellung und Fortentwicklung demokratischer Formen der Politik“. (ebd.: 33) In diesem Zusammenhang sind die Zivilgesellschaftsdiskussionen im Rahmen der osteuropäischen Dissidentenbewegungen Ende der 1970er Jahre zu nennen. „Gemeinsamer Grundzug der Debatten liegt in ihrer antitotalitären Stossrichtung“, die darauf zielt, „,den freiheitsverbürgenden Sinn der Trennung von Staat und Gesellschaft zu unterstreichen“. (ebd.: 33) Auch die sogenannten neuen sozialen Bewegungen schenken der Zivilgesellschaft erhöhte Aufmerksamkeit, da sie mit dieser ,ein Modell radikaldemokratischer Reformpolitik“ in Verbindung bringen. (Klein 2001: 33) In den westlich-demokratisch Ländern wird schon vor 1989 ein Konzept der Zivilgesellschaft entwickelt, bei dem es um einen Gegenentwurf zum Staatsinterventionismus und zur reinen Marktwirtschaft gehen sollte. Darüber hinaus stehen die neuen sozialen Bewegungen und das Konzept der Zivilgesellschaft für die Hoffnung auf eine Selbststeuerung der Gesellschaft. (Adloff 2005: 12) Trotz der unterschiedlichen Kontexte der neueren Diskurse zur Zivilgesellschaft in den 1980er Jahren ist ihnen gemeinsam, dass sie zum einen eine kritische Haltung gegenüber dem Staat offenbaren, zum anderen, ,in ein Projekt der Transformation bestehender Zivilgesellschaften münden“. (ebd.: 12) In diesem Zusammenhang können, wie Adloff überzeugend darlegt, Marx' verkürzte Interpretation der Begriffe Zivilgesellschaft bzw. bürgerliche Gesellschaft überwunden werden. Zivilgesellschaft wird dadurch als Begriff wiederentdeckt, der etwas Wünschenswertes ausdrückt und für ein ,,positives Zukunftsprojekt" steht. (ebd.: 12)

$\mathrm{Zu}$ Beginn der 1990er wird der Begriff Zivilgesellschaft in den Vereinigten Staaten breit diskutiert. Wichtig ist hier der Diskursstrang Kommunitarismus und Zivilgesellschaft. Der Kommu- 
nitarismus, ist eine politische Philosophie, die sich für die Wiederbelebung des Gemeinschaftsdenkens ,unter den Bedingungen postmoderner Informations- und Dienstleistungsgesellschaften" stark macht. (Reese-Schäfer 2001: 7; vgl. Haus 2003) Von den Kommunitarier*innen wird der Begriff Zivilgesellschaft aufgegriffen, den sie teilweise mit dem Begriff der Community synonym verwenden. (Adloff 2005: 12)

Ebenso in der Tradition des Kommunitarismus steht Robert Putnam. Ihm zufolge ist das ,Sozialkapital"12 für das Funktionieren der Zivilgesellschaft von zentraler Bedeutung. In seinem Werk „Bowling Alone“ (2000) beschäftigt er sich mit dem schwindenden Zusammenhalt in der amerikanischen Gesellschaft, für den er den Verlust an Sozialkapital verantwortlich macht. Unter Sozialkapital versteht Putnam soziale Sachverhalte wie soziale Netzwerke, Vertrauen, Werte oder Normen, die die Koordination von Handlungen ermöglichen und auf diese Weise dazu beitragen, Gesellschaft zu verbessern. (Adloff 2005: 71ff.) In den zivilgesellschaftlichen Organisationen und Vereinigungen entsteht besonders oft Sozialkapital, da die Mitglieder dort lernen, sich gegenseitig zu unterstützen, zu kooperieren und gemeinschaftsbezogen zu kommunizieren. Die Mitglieder bauen gegenseitiges Vertrauen auf und teilen gemeinsame Werte. (Putnam 2000: 20ff.) Darüber hinaus bildet sich in diesen zivilgesellschaftlichen Organisationen generalisierte Reziprozität aus, die dafür sorgt, dass die Mitglieder bereit sind, sich nicht nur gegenseitig, sondern auch nach aussen, d. h. der Gesellschaft gegenüber, Unterstützungsleistungen zur Verfügung zu stellen. (ebd.: 20) Im Hinblick auf Organisationen und Netzwerke unterscheidet Putnam zwischen abgrenzenden (bonding) und brückenbildenden (bridging) Wirkungsformen (ebd.: 22ff.) und betont, dass es vor allem die brückenbildenden Organisationen und Vereinigungen sind, denen eine besondere Bedeutung zukommt, da sie dazu beitragen, ,generalisiertes Vertrauen“ herzustellen und den gesellschaftlichen Zusammenhalt, auch unter den Bedingungen moderner Gesellschaften, zu erhalten und wiederherzustellen. (ebd.: 28) Den Verlust an Sozialkapital in den Vereinigten Staaten versucht Putnam in seinen Untersuchungen dahingehend nachzuweisen, dass er einen deutlichen Rückgang von zivilgesellschaftlichen Organisationen und Vereinigungen feststellt. (Putnam 2000) Obwohl sein methodisches Vorgehen stark kritisiert wird, erfahren seine Thesen über den Verfall des Sozialkapitals eine hohe Resonanz. Auch in Europa treffen sie auf eine positive, teilweise sehr kritische Rezeption. (Adloff 2005: 71ff.; Reese-Schäfer 2001: 107ff.; Roth 2004: 41ff.; vgl. Klein et al. 2004; Kaiser 2007) An diese Überlegungen knüpft die Enquete-Kommission „Zukunft des bürgerschaftlichen Engagements“ des Deutschen Bundestages an, die dem Begriff der Zivilgesellschaft bzw. Bürgergesellschaft grosse Bedeutung beimisst. In ihrem im Jahr 2002 vorgelegten Bericht dient ihr der Begriff als Leitbild für das bürgerschaftliche Engagement:

Die Bürgergesellschaft ist das Leitbild des bürgerschaftlichen Engagements. Bürgergesellschaft beschreibt ein Gemeinwesen, in dem die Bürgerinnen und Bürger auf der Basis gesicherter Grundrechte und im Rahmen einer politisch verfassten Demokratie durch das Engagement in selbstorganisierten Vereinigungen und durch die Nutzung von Beteiligungsmöglichkeiten die Geschicke des Gemeinwesens wesentlich prägen können.

(Enquete-Kommission ,Zukunft des bürgerschaftlichen Engagements“ 2002: 59)

\footnotetext{
${ }^{12}$ Zum Begriff Sozialkapital vgl. v. a. auch die Arbeiten von James S. Coleman (1988) bzw. soziales Kapital bei Pierre Bourdieu (1982; 1983).
} 
Bürgerschaftliches Engagement gilt, so hält die Kommission fest, als Garant für eine lebendige Zivilgesellschaft. Diese wiederum wird als Kennzeichen dafür angesehen, dass ausreichend Sozialkapital vorhanden ist, welches die Gesellschaft zusammenhält und verhindert, dass sie auseinanderdriftet. (Enquete-Kommission „Zukunft des bürgerschaftlichen Engagements“ 2002: 40) Anders als die empirischen Ergebnisse, die Putnam für die Vereinigten Staaten vorlegt, kann die Enquete-Kommission für Deutschland für den damaligen Zeitpunkt keinen Rückgang des freiwilligen Engagements feststellen. Allerdings macht der Bericht mit Verweis auf entsprechende Studien darauf aufmerksam, dass sich Motivlagen und Art des Engagements von einer längerfristigen Pflichterfüllung hin zu befristeten Projekten verändert haben. (ebd.: 49ff.; vgl. z. B. Klages/Gensicke 1999)

Ab Mitte der 1990er Jahre ist, wie Adloff (2005) bemerkt, die Verwendungsweise des Begriffs Zivilgesellschaft ,kaum noch überschaubar“ (ebd.: 12), da vermehrt Forschende, Politikverantwortliche oder Militante weltweit Bezug auf ihn nehmen und ihm immer wieder neue Bedeutungen zuschreiben. (ebd.: 12). So sehen viele den „Dritten Sektor“ als „organisierte Zivilgesellschaft“ oder sprechen von nicht-staatlichen- und non-profit-Organisationen als ,civil society organisations“. (vgl. Evers 2011: 208, Evers 2009: 79) In einer weiter gefassten Sicht ist auch von der Demokratie-fördernden Rolle der Zivilgesellschaft die Rede. Sie steht dem Staat - sozusagen autonom - gegenüber und bietet Potenziale zur demokratischen Partizipation und Demokratiebildung. Eine Zusammenarbeit von Zivilgesellschaft und Staat bzw. politischer Organisation ermöglicht es, mit den immer komplexer werdenden Herausforderungen einer globalisierten Welt umzugehen. Der Zivilgesellschaft wird zudem die Rolle als Hoffnungsträgerin in einer Zeit der Politik- und Parteienverdrossenheit zugeschrieben. (vgl. Klein 2001; Knodt/ Finke 2005) Es gibt auch Stimmen in den Debatten um den Umbau des Sozialstaates, die auf die Eigenverantwortung der Bürgerinnen und Bürger hinweisen. Immer wieder hört man von einer den Sozialstaat substituierenden Zivilgesellschaft, die Bürgerinnen und Bürger aktiv werden lässt. (Adloff 2005: 13; vgl. kritisch dazu Dahme/Wohlfahrt 2009: 241)

Angesichts zunehmender wirtschaftlicher Globalisierung kommt den Fragen hinsichtlich der Rolle der ,internationalen“ oder ,transnationalen“ Zivilgesellschaft eine wachsende politische Bedeutung zu. (Klein 2011: 33; vgl. auch Faist et al. 2014: 175ff.) Vor allem Nichtregierungsorganisationen (NGOs) und internationale globalisierungskritische Bewegungen beziehen sich auf ein Konzept internationaler Zivilgesellschaft. Das Konzept öffnet normativ den Blick auf die Entwicklung transnationaler Netzwerke, auf Prozesse der Herausbildung einer transnationalen Öffentlichkeit sowie auf die Einflussnahme der NGOs auf die politische Entscheidungsfindung. (Klein 2011: 34)

Eine weitere konzeptionelle Differenzierung des Zivilgesellschaftsbegriffs bieten Dieter Gosewinkel und andere (2004). Sie unterscheiden ein bereichslogisches Verständnis von Zivilgesellschaft von einem handlungslogischen.

Gemäss der bereichslogischen Definition bezeichnet Zivilgesellschaft den Teil der Gesellschaft, in dem sich Menschen freiwillig und im Rahmen von öffentlichen Vereinigungen für gesellschaftliche oder gemeinwohlorientiere Belange einsetzen. Zivilgesellschaft (in Deutschland wird synonym auch „Bürgergesellschaft“ verwendet) umfasst die „Gesamtheit der öffentlichen Assoziationen, Vereinigungen, Bewegungen und Verbände (...), in denen sich Bürger auf 
freiwilliger Basis versammeln und auch Einfluss auf politische Meinungsbildung nehmen“. (Klein 2011: 29) Sie weist ein breites Spektrum an Bereichen auf: von Vereinen, Stiftungen, Nachbarschaftshilfen, Initiativen und Mäzenatentum bis hin zu NGOs. (Gosewinkel et al. 2004: 11)

Nach dem bereichslogischen Konzept von Zivilgesellschaft wird diese als sozialer Bereich zwischen dem Staat, der Wirtschaft und dem privaten Bereich definiert. (Gosewinkel et al. 2004: 11) Die Assoziationen der Zivilgesellschaft befinden sich in einem ,,intermediären Raum der Öffentlichkeit“ zwischen all diesen Bereichen und sind grundsätzlich für alle offen. (Klein 2011: 29) So lassen sich auch Formen ,ungebundenen Engagements“ wie etwa Demonstrationen, Streiks oder Petitionen zur Sphäre der Zivilgesellschaft zählen, solange diese sich „durch Freiwilligkeit, Öffentlichkeit, Gemeinschaftlichkeit“ sowie durch die „Transzendierung privater Interessen“ auszeichnen. (ebd.: 29) Die Einhaltung von Menschen- und Bürgerrechten, insbesondere der staatliche Schutz der Versammlungs-, Meinungs- und Pressefreiheit, ist die Grundlage für eine lebendige Zivilgesellschaft. (Adloff 2005: 8)

Nach der handlungslogischen Definition wird Zivilgesellschaft einem ,spezifischen Typus sozialen Handelns“ zugeordnet (Gosewinkel et al.: 11): Zivilgesellschaftliche Akteurinnen und Akteure handeln erstens selbstorganisiert und selbstbestimmt (im Sinne einer gemeinsam wahrgenommenen kollektiven Selbstorganisation) und orientieren sich zweitens an Austausch und Diskussion; die Interaktionen zielen prinzipiell auf Verständigung ab. Zivilgesellschaftliche Akteurinnen und Akteure handeln zudem immer öffentlich und erkennen Vielfalt und Heterogenität an. Konflikte und Kontroversen werden drittens mit friedlichen Mitteln ausgetragen. Zivilgesellschaftliches Handeln ist also friedliches Handeln, es umschliesst aber ebenso Protestaktionen, Boykotte und zivilen Ungehorsam, sofern diese ohne Gewalt vonstattengehen. Viertens müssen sich die individuellen Motivlagen Einzelner für ein zivilgesellschaftliches Engagement, das am Gemeinwohl orientiert ist, kollektivieren lassen. (ebd.: 11) Eine handlungslogische Perspektive macht auf die besondere Handlungslogik, spezifische zivile Verhaltensweisen und Werteorientierungen in der Zivilgesellschaft aufmerksam. Hierzu gehören insbesondere Toleranz, Gewaltfreiheit, Kompromissbereitschaft sowie nicht zuletzt eine über die rein private hinausgehende Orientierung am Gemeinwesen. (Klein 2011: 29)

Adalbert Evers, der sich um ein vertieftes und differenziertes Verständnis von Zivilgesellschaft bemüht, hat in seinen Texten ein dreigliedriges Konzept entwickelt, um die verschiedenen Bedeutungszuschreibungen und Verwendungsweisen von Zivilgesellschaft möglichst klar zu erfassen. Seine Überlegungen basieren auf Michael Edwards Modell der Zivilgesellschaft (2004), in dem dieser drei Bedeutungen von Zivilgesellschaft unterscheidet. Der Begriff bezeichnet erstens das Vereinigungs-Leben, zweitens die gute Gesellschaft und drittens die Öffentlichkeit. (ebd.: 18-71) Evers' Verdienst ist es, dieses dreigliedrige Modell der Zivilgesellschaft in den deutschen Diskursraum eingebracht zu haben. Er plädiert in seinem Aufsatz ,Zivilgesellschaft, Engagement und soziale Dienste“ (2010) mit grossem Nachdruck für ein erweitertes Verständnis von Zivilgesellschaft:

Die erste, enge und am meisten vertraute Bedeutungszuschreibung meint die Fähigkeit der Gesellschaft, auBerhalb des Bereichs staatlicher Institutionen und des Marktes Assoziationen auszubilden, die als Interessen- 
verbände und Dienstleistungsträger operieren (...); hier bemisst sich dann das Ausmass, in dem eine Gesellschaft Zivil-Gesellschaft ist, vor allem an Umfang und Stärke von NGOs, Sozialprojekten, Vereinen, gemeinnützigen Organisationen und dergleichen mehr. Es gibt jedoch zwei andere, wesentlich weiter gefasste Definitionszugänge. Die erste dieser beiden Varianten diskutiert Zivilgesellschaft in Hinblick auf die Relevanz zivilgesellschaftlicher Werte für die Gesellschaft insgesamt - also auch für staatliches Handeln und das Handeln von Wirtschaftsunternehmen. Welche Geltung haben hier Normen zivilen Verhaltens wie wechselseitiger Respekt, Gemeinsinn, Dialog- und Kooperationsfähigkeit (...)? Eine andere weite Variante spricht von Zivilgesellschaft, wenn sie Gesellschaft nicht so sehr als soziales Gebilde, sondern als politisches Gemeinwesen versteht - also als Bürgergesellschaft und demokratische Republik. Ihre Stärke ist die Ausbildung einer politischen Öffentlichkeit (...). Dieser Blick auf die Bürgergesellschaft unterläuft die übliche Annahme, dass Politik mit staatlichem Handeln gleichzusetzen ist. (2010: 282f.)

Auch Frank Adloff (2005) verortet Zivilgesellschaft konzeptionell in verschiedenen Bereichen. Der Begriff hat für ihn drei Bedeutungen. Er bezeichnet

einen gesellschaftlichen Bereich von Organisationen und Institutionen, zivile Umgangsformen und ein utopisches Projekt. Zivilgesellschaft vermittelt zwischen Staat und Gesellschaft, ist also ein Konzept, das den Politikbegriff weit fasst und nicht nur auf staatliches Handeln festlegt, gleichzeitig aber durch ein schärferes konturiertes Verständnis von Gesellschaft gekennzeichnet ist. Denn während üblicherweise unter Gesellschaft im Grunde alles verstanden wird, was sich innerhalb von Nationalstaaten abspielt: Familien, Wirtschaft, Recht, Politik, Öffentlichkeit, Wissenschaft usw., meint Zivilgesellschaft allein die freiwilligen und öffentlichen Vereinigungen von Bürgern und Bürgerinnen. (ebd.: 9)

Die verschiedenen Konzepte und Modelle von Zivilgesellschaft, die ich in diesem Kapitel vorgestellt habe, machen deutlich, dass sie normativ ausgerichtet sind. Diese normative Orientierung ist in den Diskursen zur Zivilgesellschaft sehr präsent und ein Stück weit typisch für diese. Der Begriff in seiner normativen Lesart verweist immer auch auf ,empathische Erwartungen“ (Bode et al. 2009: 8), die ihn dann besonders anfällig für Ideologien macht. Dies zeigt sich besonders in den mit ihm assoziierten Begriffen wie „Gemeinwohl“" oder „Gemeinschaft“, die das Verständnis der Zivilgesellschaft rasch in Richtung moralisierender Diskurse (ebd.: 8) oder „neuautoritäre[r] Fundamentalismen“ (Reese-Schäfer 2001: 7) drängen.

\subsection{Diskussionen über das freiwillige gesellschaftliche Engagement}

Innerhalb der aktuellen wissenschaftlichen Diskussionen zum freiwilligen, zivilgesellschaftlichen Engagement finden sich in Deutschland und der Schweiz verschiedene Begriffe, die mit unterschiedlichen Bedeutungen konnotiert sind. So ist von „Freiwilligkeit“ oder „Freiwilligenarbeit“ die Rede, aber auch von „freiwilligem Engagement“, „,ürgerschaftlichem Engagement“ oder „Ehrenamt“. Und schliesslich tauchen Konzepte wie „Bürger- oder Zivilgesellschaft“ „soziales Kapital“ auf, die in der Diskussion um freiwilliges bzw. zivilgesellschaftliches Engagement eine prominente Rolle spielen. Doch wie werden diese Begriffe verwendet und mit welchen Diskursen sind sie belegt?

So stösst man im deutschen Diskurs immer wieder auf die Begriffe Ehrenamt und freiwilliges Engagement, die sowohl im Alltag freiwillig Engagierter, aber auch in der Fachdiskussion benutzt werden. (Olk/Hartnuss 2011: 145f.; Gensicke et al. 2006; 2010; Simonson et al. 2016) Im Kern wird Ehrenamt als „Synonym für eine klassische und historisch gewachsene Form gemeinwohlorientierten Tätigkeiten“ (Strickler 2011: 163) begriffen. In der Fachdiskussion ist die Bezeichnung Ehrenamt solchen Tätigkeiten vorbehalten, für die man berufen oder gewählt 
wird. In seinem alltagssprachlichen Gebrauch jedoch ist dieses Wort ,als Oberbegriff für alle Ausdrucksformen des freiwilligen, unentgeltlichen und gemeinwohlorientierten Engagements“ (Olk/Hartnuss 2011: 146) üblich. Dabei ist weder ein gewähltes Amt noch eine öffentliche Ehrerweisung impliziert. (ebd.: 146)

Auch für den Begriff freiwilliges Engagement gibt es verschiedene Verwendungsweisen. Er betont die Freiwilligkeit des Engagements. Das Engagement als solches erscheint hier als ,eine selbst gewählte Aktivität, die den subjektiven Bedürfnissen, Interessen und Sinnorientierungen der Individuen entspricht und deshalb als Ausdruck eines individuellen Lebensstils“ (Olk/Hartnuss 2011: 146) in Abgrenzung zu Formen der Pflichterfüllung ausgeübt wird. Im deutschen Freiwilligensurvey (vgl. Gensicke et al. 2006; 2010; Simonson et al. 2016) stützt sich der Begriff des freiwilligen Engagements hingegen auf eine „operationale Definition“. (Olk/Hartnuss 2011: 149)

Für den Freiwilligensurvey in Deutschland gelten die gleichen Definitionskriterien wie für die Enquete-Kommission „Zukunft des bürgerschaftlichen Engagements“ (2002). Im Bericht der Enquete-Kommission ist allerdings nicht von freiwilligem, sondern von bürgerschaftlichem Engagement die Rede. Der Begriff des bürgerschaftlichen Engagements ist mittlerweile in der Politik und öffentlichen Diskussion in Deutschland etabliert. Dazu beigetragen haben insbesondere die Arbeiten der Enquete-Kommission ,Zukunft des bürgerschaftlichen Engagements“ des Deutschen Bundestags und der in der Folge entstandenen Institutionen (Unterausschuss „Bürgerschaftliches Engagement“ des Deutschen Bundestags und „Bundesnetzwerk Bürgerschaftliches Engagement") (2002). Die Enquete-Kommission legt in ihrem Bericht eine Definition von bürgerschaftlichem Engagement vor, die durch folgende Kriterien gekennzeichnet ist: Zunächst handelt es sich um eine Tätigkeit, die aus freiem Willen und aufgrund der eigenen Motivation verrichtet wird und nicht gewinnorientiert ist. Ferner geht es um den Einsatz für Dritte bzw. für das Gemeinwesen. Dieser ist im Unterschied zur Haus- und Familienarbeit im öffentlichen Raum situiert, und die Tätigkeit wird gemeinschaftlich/kooperativ ausgeübt. (ebd.: 38) Im Unterschied zum Begriff des freiwilligen Engagements ,handelt es sich bei dem Begriff des ,bürgerschaftlichen Engagements' um keine rein analytische Kategorie, sondern immer auch um eine normativ-programmatische Leitformel“. (Olk 2002: 29; vgl. auch Roth 2000: 31f.) In der Schweiz ist der Terminus des bürgerschaftlichen Engagements weniger gebräuchlich und eher selten anzutreffen. Stattdessen wird in der Fachdebatte vom, freiwilligen Engagement " gesprochen. In der deutschsprachigen Schweiz wird Freiwilligenarbeit vor allem als Oberbegriff für verschiedene Formen des Engagements verwendet, in der französischen Schweiz ist entsprechend von bénévolat die Rede. (Nadai 2004: 19) Freiwilligkeit geht über das Phänomen Freiwilligenarbeit ein gutes Stück hinaus und umfasst noch andere Formen, sich für die Allgemeinheit einzusetzen. Hierzu gehört der Einsatz von Prestige für gemeinnützige Anliegen und die Weitergabe von Geld oder Naturalien. (vgl. Ammann 2001: 17; Stadelmann-Steffen et al. 2010: 29)

Obgleich die Definitionen von Freiwilligenarbeit variieren, lassen sich laut dem FreiwilligenMonitor der Schweiz als gemeinsamer Kern vier Elemente identifizieren, die denen des wissenschaftlichen Diskurses innerhalb Deutschlands sehr ähnlich sind: Es handelt sich dabei um 
eine Tätigkeit, die aus freiem Willen und aufgrund der eigenen Motivation verrichtet wird. Darüber hinaus wird Freiwilligenarbeit unbezahlt geleistet. Ferner handelt es sich bei der Freiwilligenarbeit um einen Einsatz für Dritte bzw. für das Gemeinwesen. Und schließlich wird Freiwilligenarbeit im Unterschied zur Haus- oder Familienarbeit ausserhalb des eigenen Haushalts ausgeübt, in der Regel im Kontext einer Organisation oder im privaten und nachbarschaftlichen Bereich ausserhalb der eigenen vier Wände resp. dem familiären Umfeld. (Freitag et al. 2016: 33f.)

Grundsätzlich lässt sich zwischen formeller und informeller freiwilliger Tätigkeit unterscheiden. (Freitag et al. 2016: 34ff.) Informelle freiwillige Tätigkeiten finden ohne festen organisationalen Rahmen statt und beruhen auf individueller Initiative. Dazu gehört beispielsweise das Hüten fremder Kinder, Einkaufen für andere, Transporte von Personen mit Einschränkungen etc., also unbezahlte Tätigkeiten ausserhalb des eigenen Haushalts im Rahmen von verwandtschaftlichen, freundschaftlichen oder nachbarschaftlichen Netzwerken. Unter formellen Tätigkeiten werden freiwillige Arbeiten im Rahmen von Organisationen, Vereinen und Institutionen verstanden. Sitzungsgelder, Spesenvergütungen oder symbolische Beiträge gelten hier nicht als Bezahlung. Diese Tätigkeiten können nach dem Verpflichtungsgrad weiter unterschieden werden: Ehrenamtlich tätige Personen sind in der Regel gewählt (Vorstand in einem Verein etc.); ihr Engagement ist verpflichtender als die „gewöhnliche“ formelle freiwillige Tätigkeit wie beispielsweise der Einsatz als volunteer bei einem grossen Sportanlass oder Helfertätigkeiten bei einem Vereinsfest. (ebd.: 34)

Weiterhin findet in der Schweiz auch der Begriff des Ehrenamts Verwendung. Selbst wenn im Alltag der Engagierten häufig keine Differenzierung zwischen Ehrenamt und Freiwilligenarbeit vorhanden ist, so ist in der Fachdiskussion die Bezeichnung als Ehrenamt solchen Tätigkeiten vorbehalten, für die man berufen oder gewählt wird und mit denen Führungs- und Repräsentationsfunktionen verbunden sind. (Nadai 2004: 26; Freitag et al. 2016: 34ff.) Das folgende Schaubild verdeutlicht die im Schweizer Diskurs unterschiedenen Bereiche der Freiwilligkeit:

Abbildung 7: Bereiche der Freiwilligkeit in der Schweiz

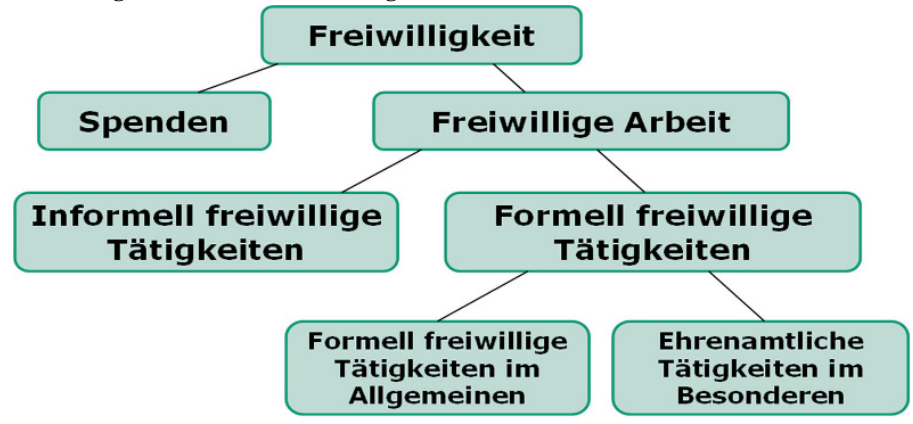

Quelle: Eigene Darstellung in Anlehnung an Freitag et al. 2016: 34ff.

In Deutschland ist der Begriff der Freiwilligenarbeit hingegen wenig geläufig. Hier bringt der Begriff des freiwilligen Engagements ,alte“ und „neue“ Formen des Engagements auf einen 
einheitlichen Begriff. Denn das Ehrenamt verändert sich stetig. Daher muss es in engem Zusammenhang mit den gesellschaftlichen Rahmenbedingungen und deren Wandel über die Zeit gesehen werden. Kurz gesagt: Es gibt eine breite Vielfalt von Engagementformen. Das „alte“ Ehrenamt ist meist in überkommene Sozialmilieus eingebunden, hoch organisiert und unterliegt den Weisungen professioneller Mitarbeiter. Im Unterschied dazu ist das „neue“ Ehrenamt eher durch die Erfahrung konkreter Benachteiligungen und Leiden motiviert, entwickelt sich in überschaubaren lokalen Zusammenhängen und findet in weitgehend selbstbestimmten, autonomen und gering formalisierten Organisationsformen statt. (vgl. Olk 1989; Beher et al. 2000; Röbke 2011)

In seiner normativen Dimension verweist der Begriff des bürgerschaftlichen resp. freiwilligen Engagements, wie früher bereits dargelegt, auf das Konzept der „Bürgergesellschaft“ (in Deutschland) bzw. der ,Zivilgesellschaft“. Damit wird ein Gemeinwesen beschrieben, ,in dem die Bürgerinnen und Bürger auf der Basis gesicherter Grundrechte und im Rahmen einer politisch verfassten Demokratie durch das Engagement in selbstorganisierten Vereinigungen und durch die Nutzung von Beteiligungsmöglichkeiten die Geschicke des Gemeinwesens wesentlich prägen können.“ (Enquete-Kommission 2002: 59) Wie Eva Nadai feststellt, wird die Diskussion um die Bürgergesellschaft vor allem in Deutschland geführt und hat für die Schweiz weniger Relevanz. (2004: 27)

Dagegen schliessen Fachdiskussionen in der Schweiz ebenso wie in Deutschland an den Begriff des ,sozialen Kapitals“ an. (vgl. z. B. Bühlmann/Freitag 2007; Klein et al. 2004; StadelmannSteffen et al. 2007, 2010; Freitag 2014; Freitag et al. 2016) Einblick in verschiedene Facetten des Sozialkapitals gibt insbesondere das von Markus Freitag (2014) herausgegebene Werk „Das soziale Kapital der Schweiz“, in dem die mobilisierende Wirkung sozialen Kapitals auf freiwilliges Engagement (egal ob formell oder informell) hervorgehoben wird. Freitag versteht unter Sozialkapital:

die Vorteile, die sich aus der jeweils vorhandenen Sozialstruktur ergeben und deren Wert sich in der erfolgreichen Umsetzung selbst vorgegebener Ziele des Individuums oder ganzer Gruppen und Gemeinschaften zeigt. Ohne mögliche negative Folgen von Sozialstrukturen zu verneinen, gehen wir davon aus, dass sowohl ein Engagement in Vereinen, die unbezahlte Arbeit für die Gemeinschaft, die Hilfeleistungen im sozialen Umfeld von Familie, Freunden, Kollegen und Nachbarn als auch ein Zutrauen in das Gegenüber, die Unterstützung von Normen reziproker Handlungen und tolerante Einstellungen nicht nur für die Gemeinschaft, sondern auch für das Individuum selbst nützlich sind. (ebd.: 31 )

Basierend auf den bereits erwähnten Überlegungen von Tocqueville (1835/40) und Putnam (2000) sehen Freitag und Ackermann (2014) im freiwilligen Engagement in Vereinen eine entscheidende Rolle bei der Bildung von sozialem Kapital. Ihrer Auffassung nach gehört in der Schweiz „das soziale Miteinander in Vereinen neben dem zwischenmenschlichen Vertrauen zur Basiskategorie der modernen Sozialkapitalkonzeption“. (ebd.: 78)

In meiner Studie verwende ich den Terminus ,freiwilliges Engagement“ in Anlehnung an die Definition des Schweizer Freiwilligen-Monitors, welcher zwischen formeller freiwilliger Tätigkeit und informeller freiwilliger Tätigkeit unterscheidet. (Freitag et al. 2016) Meiner Meinung nach sind beide Formen der Freiwilligentätigkeit von Bedeutung. 


\subsection{Empirische Befunde zum freiwilligen Engagement in der Schweiz}

Für den Untersuchungsgegenstand meiner Arbeit sind empirische Studien zum freiwilligen Engagement im Allgemeinen und im Speziellen zum Engagement der sogenannten „,neuen Zugewanderten“ (vgl. Baghdadi 2010; Müller-Jentsch/Avenir Suisse 2008) in der Schweiz relevant. In diesem Kapitel werde ich auf die Datenlage zum freiwilligen Engagement in der Schweiz und ergänzend in Deutschland eingehen. Im Vordergrund stehen die Ergebnisse des Freiwilligen-Monitors der Schweiz (Freitag et al. 2016) und des Freiwilligensurveys in Deutschland (Simonson et al. 2016). Beide Studien basieren auf repräsentativen Umfragen, wurden bereits in mehreren Erhebungswellen durchgeführt und haben sich in ihren jeweiligen Ländern etabliert. Zuerst werde ich zeigen, wie sich aktuell die Engagementquoten beider Länder darstellen bzw. wie sie sich (bis zur letzten Erhebung) entwickelt haben. Weiter werde ich die Daten zu Motiven und Motivlagen für freiwilliges Engagement in den beiden Ländern diskutieren sowie den Blick auf die regionalen Unterschiede im Engagement richten, um dann die Situation der Forschung zum Engagement in Vereinen zusammenzufassen. Das Kapitel schliesst mit einer Darstellung der bisher vorliegenden Befunde zum Engagement der neuen Zugewanderten in der Schweiz.

\subsubsection{Aktuelle Bestandsaufnahmen zum freiwilligen Engagement}

Die Datenlage zum freiwilligen Engagement im Allgemeinen ist meiner Ansicht nach für die Schweiz und Deutschland sehr gut. Für die Schweiz gibt aktuell insbesondere der FreiwilligenMonitor (vgl. Freitag et al. 2016) Auskunft. Er stützt sich auf Daten, welche das Bundesamt für Statistik (BfS) im Rahmen der regelmässigen Schweizerischen Arbeitskräfteerhebung (SAKE) erhebt. In Deutschland kann vor allem auf den Freiwilligensurvey (vgl. Simonson et al. 2016) zurückgegriffen werden. Problematisch ist jedoch die fehlende Vergleichbarkeit der genannten Studien, da die verschiedenen Erhebungen mit unterschiedlichen Engagementbegriffen und definitionen operieren und jeweils unterschiedliche Altersgruppen bilden.

Der erste Freiwilligen-Monitor der Schweiz erschien im Jahr 2007. Er gab einen umfassenden Einblick in Ausmass, regionale Unterschiede und Motive des freiwilligen Engagements. (vgl. Stadelmann-Steffen et al. 2007) Von Beginn an war geplant, dass regelmässig alle vier oder fünf Jahre erneute Erhebungen durchgeführt werden. (vgl. Ammann 2007: 11) Im Jahr 2010 folgte der zweite Freiwilligen-Monitor, der auf der Datengrundlage des Jahres 2009 erstellt wurde. (vgl. Stadelmann-Steffen et al. 2010) Aktuell ${ }^{13}$ liegt der dritte Freiwilligen-Monitor vor, der auf Daten aus dem Jahr 2014 basiert. (vgl. Freitag et al. 2016)

Der Schweizer Freiwilligen-Monitor unterscheidet, wie ich schon früher betont habe, drei Formen der Freiwilligkeit:

„(1) Freiwillige Tätigkeiten, die innerhalb von Vereins- oder Organisationsstrukturen ausgeübt

\footnotetext{
${ }^{13}$ Im Frühjahr 2019 wurden die Erhebungen zum vierten Freiwilligen-Monitor der Schweiz durchgeführt. Die Publikation folgt im Jahr 2020. (Schweizerische Gemeinnützige Gesellschaft 2019)
} 
werden (formelle Freiwilligkeit), (2) freiwillige Arbeiten wie Nachbarschaftshilfe oder das Hüten fremder Kinder, die ausserhalb solcher Organisationsstrukturen stattfinden (informelle Freiwilligkeit) sowie (3) Spenden von Geld oder Naturalien“. (Freitag et al. 2016: 15)

Damit wird hier eine andere Differenzierung vorgenommen als im deutschen Freiwilligensurvey, der zwischen Gemeinschaftsaktivitäten bzw. öffentlicher Aktivität und freiwilligem Engagement unterscheidet. (Simonson et al. 2016: 15ff.) Ferner erfasst der Freiwilligen-Monitor im Unterschied zum Freiwilligensurvey auch monetäre Leistungen ${ }^{14}$.

\subsubsection{Höhe und Entwicklung von Quoten des freiwilligen Engagements}

Der Freiwilligen-Monitor 2016 stellt fest, dass rund ein Viertel der schweizerischen ständigen Wohnbevölkerung über 15 Jahren im Sinne einer formellen Freiwilligkeit innerhalb von Vereinsstrukturen engagiert ist. 38 Prozent der Bevölkerung sind im Sinne einer informellen Freiwilligkeit, d. h. ausserhalb von Organisationen, engagiert. (Freitag et al. 2016: 16)

Vergleicht man die Daten aus dem Jahr 2014 mit den früheren Erhebungswellen in den Jahren 2006 und 2009, so fällt auf, dass die formelle Freiwilligkeit in der Schweiz leicht rückläufig ist (2006: $28 \%$, 2009: $26 \%$, 2014: $25 \%$ ) (ebd.: 49), während die informelle Freiwilligkeit nach einem Rückgang im Jahr 2009 wieder deutlich angestiegen ist (2006: 37 \%, 2009: 29 \%, 2014: $38 \%$ ). (ebd.: 77 )

Für den deutschen Freiwilligensurvey liegen inzwischen Ergebnisberichte der ersten vier Erhebungswellen (1999, 2004, 2009, 2014) vor. (vgl. Rosenbladt 2000; Gensicke et al. 2006, 2010; Simonson et al. 2016) Die Ergebnisse der Erhebung von 2014 wurden 2016 veröffentlicht ${ }^{15}$. Die Untersuchungen legen offen, dass die Engagementquote, d. h. der Anteil der deutschen Wohnbevölkerung ab 14 Jahren, der sich freiwillig engagiert, zwischen 1999 und 2014 stark gestiegen ist (1999: $34 \%$, 2004: $36 \%$, 2010: $36 \%$, 2014: $44 \%$ ). (Simonson et al. 2016: 15f.) 70 Prozent der Befragten waren 2014 in Deutschland zudem öffentlich, d. h. ausserhalb von Beruf und Familie in irgendeiner Form gemeinschaftlich aktiv. (ebd.: 16f.)

Im Schweizer Freiwilligen-Monitor sowie im deutschen Freiwilligensurvey zeigt sich zudem, dass eine hohe Bildung, eine gesicherte finanzielle Situation und eine gute berufliche Stellung die Wahrscheinlichkeit eines freiwilligen Engagements erhöhen. (Freitag et al. 2016: 66; Simonson et al. 2016: 19)

\subsubsection{Motive und Motivlagen für freiwilliges Engagement}

In Bezug auf die Motive des freiwilligen Engagements gibt es keine bedeutenden Unterschiede zwischen den beiden Ländern. (Freitag et al. 2016: 93ff.; Simonson et al. 2016: 115ff.) Der aktuelle Freiwilligen-Monitor Schweiz 2016 (Freitag et al. 2016) hat neben den Motiven der

\footnotetext{
${ }^{14}$ Seit der dritten Erhebungswelle erfasst der Freiwilligen-Monitor auch erstmals die „Freiwilligkeit im Internet“. Darunter fallen laut Definition des Freiwilligen-Monitors ,,das Gründen und Moderieren von Facebook-Gruppen oder die Pflege von Webseiten von Vereinen und Organisationen“. (Freitag et al. 2016: 18)

${ }^{15}$ Die Ergebnisse des fünften Freiwilligensurveys 2019 werden voraussichtlich Ende 2020 veröffentlicht. (Deutsches Zentrum für Altersfragen 2019)
} 
formell Freiwilligen auch jene der informell Tätigen erhoben und dabei interessante Unterschiede festgestellt. Für Letztere steht vor allem die persönliche Hilfestellung (,Anderen Menschen helfen“) im Vordergrund. „Der Akt der Gemeinsamkeit (,Mit Anderen etwas bewegen“) und die Möglichkeit, sich weiterzuentwickeln (,Eigene Kenntnisse und Erfahrungen erweitern', ,Sich persönlich weiterzuentwicklen'), kennzeichnen in besonderer Weise das formelle Engagement und heben den Stellenwert von Vereinen und Organisationen als Schulen der persönlichen Entwicklung und des sozialen Miteinanders in strukturierten und regelmässig abgerufenen Beziehungsgeflechten besonders hervor.“ (ebd.: 95) Das freiwillige Engagement in der Schweiz zeichnet sich, wie Freitag et al. prägnant formulieren, insgesamt als eine „Mischung aus altruistischen und stärker selbstbezogenen Beweggründen“ (ebd.: 17) aus.

Der Freiwilligensurvey hat für Deutschland eine ähnliche Motivlage für freiwilliges Engagement ermittelt. Dort wird ein Mix aus gesellschaftlichen, sozialen und persönlichen Motiven beobachtet, die für die Motivation zum freiwilligen Engagement wichtig sind. (Simonson et al. 2016: 418ff.) Spass und Freude an der Tätigkeit ist und bleibt im Freiwilligensurvey die „oberste Maxime“ für freiwilliges Engagement. Es engagieren sich aber ebenfalls viele, um mit anderen Menschen zusammenzukommen oder um die Gesellschaft mitzugestalten. (ebd.: 418) Weit weniger häufig werden Motive genannt, die einem Status- oder beruflichen Gewinn dienen. Nur vergleichsweise wenige wollen durch ihr Engagement „Ansehen und Einfluss gewinnen“, „,beruflich vorankommen“, „etwas dazuverdienen“ oder „Qualifikationen erwerben“. (ebd.: 419) Zu vergleichbaren Ergebnissen kommt die Allensbach-Studie zu den Motiven bürgerschaftlichen Engagements in Deutschland (BMFSFJ 2014). Auch hier spielen für den Grossteil der Engagierten neben altruistischen Motiven selbstbezogene Antriebe für ein Engagement eine Rolle. (ebd.: 3) Die Ergebnisse des Freiwilligensurveys zeigen, wie ich hinzufügen möchte, dass das Engagement als „Lernfeld“, in dem man eigene Kenntnisse und Erfahrungen erweitern, aber auch eigene Kenntnisse einbringen kann, wichtiger wird. (Gensicke et al. 2010: 119)

\subsubsection{Regionale Unterschiede im freiwilligen Engagement}

In beiden Ländern sind regionale Unterschiede im Engagement zu finden. Für die Schweiz hat der Freiwilligen-Monitor (Freitag et al. 2016) festgestellt, dass in der deutschsprachigen Schweiz viel mehr Menschen sowohl formell als auch informell freiwillig tätig sind als dies in der Suisse romande und der italienischsprachigen Schweiz der Fall ist. (ebd.: 71) Während in der deutschsprachigen Schweiz beinahe ein Drittel der Wohnbevölkerung (29\%) formell in Vereinen und Organisationen freiwillig engagiert sind, tut dies in der lateinischen Schweiz weniger als ein Fünftel (jeweils $17 \%$ in der Suisse romande und der italienischsprachigen Schweiz). (ebd.: 71) Informelles Engagement ausserhalb von Vereinen und Organisationen ist innerhalb der Landesteile ebenso ungleich verteilt. So ist der Anteil informell Freiwilliger in der deutschsprachigen Schweiz mit 41 Prozent signifikant höher als in der Romandie (mit $30 \%$ ).

Für Deutschland stellt der aktuelle Freiwilligensurvey ebenfalls regionale Unterschiede im freiwilligen Engagement fest. (Simonson et al. 2016: 21) Freiwilliges Engagement ist in deutschen 
ländlichen Gebieten stärker vertreten als in städtischen Regionen. In Grossstädten ist das Engagement am niedrigsten. In Regionen mit hoher Arbeitslosigkeit ist der Anteil freiwillig Engagierter geringer als in Regionen mit niedriger Arbeitslosigkeit. Grosse Unterschiede gibt es weiterhin zwischen den alten und neuen Bundesländern. In Ostdeutschland (mit 39\%) sind immer noch deutlich weniger Menschen freiwillig engagiert als in Westdeutschland (45\%). Der Freiwilligensurvey zeigt aber darüber hinaus, dass das Engagement in den neuen Bundesländern (und Berlin) seit 1999 stark gestiegen ist. (ebd.: 21)

\subsection{Vereine als Orte des freiwilligen Engagements der Schweiz}

Vereine und Freiwilligenorganisationen bilden die Grundpfeiler des freiwilligen Engagements. Sie sind Orte zum gegenseitigen Austausch und zur Zusammenarbeit und leisten einen wichtigen Beitrag für den gesellschaftlichen Zusammenhalt. (vgl. Freitag 2014; Bühlmann/Freitag 2007; Putnam 2000) Laut dem vielzitierten Schweizer Freiwilligen-Monitor sind Vereine „Orte, die den Menschen Gelegenheit zu gegenseitigem Austausch und zur Zusammenarbeit bieten. Damit stellen sie eine wichtige Form von ,Sozialkapital' dar und haben eine herausragende Bedeutung für den gesellschaftlichen Zusammenhalt“". (Stadelmann-Steffen et al. 2010: 163) Dieser gesellschaftliche Zusammenhalt wird umso besser gelingen, je mehr persönliche Begegnungen von Menschen aus unterschiedlichen gesellschaftlichen Bereichen in den Vereinen stattfinden. (ebd.: 163) „Der Verein als Hort des sozialen Miteinanders“ ist nach Freitag und Ackermann (2014) ,ein zentraler Baustein der Sozialkapitaltheorie“. (ebd.: 41) In ihrem Aufsatz „Vereinsengagement als soziales Kapital der Schweiz“ merken sie zu Recht an, dass „Vereine als ,Schulen der Demokratie“ zur politischen Sozialisation beitragen, da sie in der Praxis die Verbindung von Mitgliedschaft- und Staatsbürgerrolle ermöglichen und bei ihren Mitgliedern die Grundregeln demokratischen Verhaltens einstudieren“. (ebd.: 41f.)

Dieser Blick auf Vereine und Organisationen knüpft, wie früher schon erwähnt, an Robert Putnam (2000) an, welcher Vereine als Orte generalisierender Reziprozität betrachtet. Die einzelnen Mitglieder lernen dort zum einen, sich gegenseitig zu unterstützen, zu kooperieren; sie bauen gegenseitiges Vertrauen auf und teilen gemeinsame Werte. Zum andern sind sie aufgrund dieser Erfahrungen eher bereit, sich nicht nur innerhalb des Vereins, sondern auch nach aussen, d. h. der Gesellschaft gegenüber, zu engagieren. (Putnam/Goss 2001: 21ff.) Überdies unterscheidet Putnam zwischen brückenbildenden und abgrenzenden Vereinigungen: Brückenbildende Vereine zeichnen sich dadurch aus, dass sie Menschen mit unterschiedlichen sozialen Merkmalen und Hintergründen zusammenbringen und auf diese Weise „Brücken“ zwischen ihnen bauen. $\mathrm{Zu}$ denken wäre hierbei an einen Sportverein oder Chor, in denen Menschen aus den unterschiedlichen Berufen und kulturellen Kontexten miteinander ihre Freizeit verbringen und sich so näherkommen können. Abgrenzende Vereine setzen sich dagegen nur aus Personen zusammen, die einander sehr ähnlich sind, da sie durch eine gemeinsame Herkunft einander verpflichtet sind oder ein bestimmtes Merkmal teilen. Durch gemeinsame Aktivitäten haben sie einen gemeinsamen Erfahrungshorizont, der gemeinschafts- und gruppenstiftend wirkt. Als Beispiel seien hier folgende Vereinigungen und Gruppen angeführt: Frauengruppen oder Seni- 
orenvereinigungen, aber auch berufliche Interessenverbände, in denen sich Menschen des gleichen Berufs organisieren, oder politische Parteien. (Putnam/Goss 2001: 28ff.) Mit Blick auf den gesamtgesellschaftlichen Zusammenhalt fallen vor allem brückenbildende, zwischen verschiedenen gesellschaftlichen Bereichen vermittelnde Vereinigungen ins Gewicht. Hier kommen Menschen zusammen, die üblicherweise sehr wenig in Kontakt stehen und erfahren sich als Gleichgesinnte. Die abgrenzenden Vereinigungen hingegen fördern aufgrund starker Identifikationspotenziale den Zusammenhalt innerhalb der Gruppe und haben keine grosse Ausstrahlungskraft nach aussen. Da dieser Vereinigungstyp meist nur eine lockere Verbindung zur restlichen Gesellschaft (d. h. ausserhalb des Vereins) aufrechterhält, trägt er zum gesamtgesellschaftlichen Zusammenhalt und zur Stärkung der zivilgesellschaftlichen Partizipation nur wenig bei. (Stadelmann-Steffen et al. 2010: 163f.) In der Praxis ist diese Unterscheidung allerdings nicht immer einfach zu treffen. Denn viele Vereine und Organisationen sind abgrenzend und brückenbildend zugleich. Schon Putnam hat bemerkt, dass es sich bei der Unterscheidung zwischen brückenbildend und abgrenzend weniger um ein eindeutiges Entweder-Oder als vielmehr um ein graduelles Merkmal von Freiwilligenorganisationen handelt. (Putnam/Goss 2001: 29; vgl. Freitag/Ackermann 2014: 45)

Doch wie steht es nun genau um das Vereinsengagement in der Schweiz? In welchen Bereichen engagieren sich die Menschen? Diese Fragen lassen sich mit den Daten des letzten FreiwilligenMonitors aus dem Jahre 2014 beantworten. Mit 30 Prozent der Wohnbevölkerung zählen Sportvereine weitaus am meisten Mitglieder. An zweiter Stelle folgen kirchliche Organisationen und Interessenverbände (je rund $20 \%$ ). An dritter Stelle finden sich Spiel,- Hobby- und Freizeitvereine (je rund $19 \%$ ). Kulturelle und soziale/karitative Vereine (je $13 \%$ ) rangieren hingegen erst auf dem vierten Platz. Betrachtet man lediglich jene Mitglieder, die tatsächlich aktiv am Vereinsleben teilnehmen, beteiligt sich die Wohnbevölkerung am häufigsten in Sportvereinen (26\%), gefolgt von Spiel-, Hobby- und Freizeitvereinen (15\%) sowie kirchlichen Organisationen (12\%). Freitag et al. (2016) gehen in ihrer Analyse einen Schritt weiter und nehmen eine Differenzierung der formellen Freiwilligenarbeit nach Vereinstypen vor. Innerhalb von Organisationen und Vereinen unterscheiden sie zwischen formell freiwilligen Tätigkeiten und ehrenamtlichen Tätigkeiten. Formelle Freiwilligenarbeit wird besonders häufig in Sportvereinen ausgeführt (12\%), am zweithäufigsten in Spiel-, Hobby- und Freizeitvereinen (8 \%). Das gleiche Muster lässt sich bei der Ausübung eines Ehrenamtes ausmachen: Ehrenamtlich Tätige finden sich am ehesten in einem Sportverein (5\%) oder Spiel-, Hobby- und Freizeitverein (3\%). (ebd.: 51f.)

Der Vergleich zum Freiwilligensurvey zeigt, dass in Deutschland die Verteilung der Engagement- und Aktivitätsquoten nach gesellschaftlichen Bereichen ein ähnliches Bild aufweist. So sind es dort ebenfalls die Bereiche Sport und Bewegung, in denen sich die Menschen am häufigsten freiwillig engagieren (16\%) oder gemeinschaftlich aktiv sind (27\%). Danach folgen die Bereiche Schule oder Kindergarten (dort sind $5 \%$ gemeinschaftlich aktiv oder $9 \%$ freiwillig engagiert), Kultur und Musik (mit einer Aktivitätsquote von $10 \%$ beziehungsweise einer Engagementquote von $9 \%$ ), Soziales (mit einer Aktivitätsquote von $7 \%$ beziehungsweise einer Engagementquote von $9 \%$ ) sowie der kirchliche oder religiöse Bereich (mit einer Aktivitätsquote von $5 \%$ beziehungsweise einer Engagementquote von $8 \%$ ). (Simonson et al. 2016: 110) 
Die Verteilung von Aktivitäten und Engagement in den verschiedenen gesellschaftlichen Bereichen beider Länder verdeutlicht, dass ein Grossteil des aktiven Vereinslebens in Bereichen stattfindet, die in Anlehnung an Putnam (2000) eher einen brückenbildenden Charakter haben. Sport- Freizeit-, Musik- und Kulturvereine vereinen Menschen mit unterschiedlichen sozialen Hintergründen zur Verfolgung eines bestimmen Zwecks: Man treibt zusammen Sport, singt in einem Chor oder spielt zusammen Theater. Es sind Putnam zufolge die Vereine mit brückenbildenden Effekten, die den gesellschaftlichen Zusammenhalt und die zivilgesellschaftliche Einbindung fördern. Ihnen gelingt es, Menschen unterschiedlicher Herkunft zusammenzubringen. (vgl. Freitag/Ackermann 2014: 45) Die Befunde aus beiden Surveys lassen sich dahingehend deuten, dass der gesellschaftliche Zusammenhalt in der Schweiz und in Deutschland stark und grösser als weithin vermutet ist. Eine weitere Folgerung ist: In der Schweiz und in Deutschland lernen viele Menschen durch eine gesellschaftliche Aktivität oder ein freiwilliges Engagement in einem Verein sich zivilgesellschaftlich zu betätigen, obwohl sie sich (noch) nicht als Teil eines gesellschaftlichen Ganzen begreifen. So entsteht eine zivilgesellschaftliche Infrastruktur (vgl. Kap. 5.1 meiner Arbeit), in der sich eine Vielzahl aktiver und engagierter Menschen in brückenbildenden Vereinigungen und Organisationen zusammenschliesst.

Damit ist ein Sachverhalt angesprochen, der für meine Arbeit von grossem Interesse ist, da mich hier das Thema des freiwilligen Engagements von Expatriates besonders beschäftigt. Doch lassen sich Engagement und Sozialkapital immer in einem Zusammenhang denken, der zwingend einen gesellschaftlichen Zusammenhalt und die zivilgesellschaftliche Einbindung fördert? Zur Erörterung dieser Frage werde ich an eine Diskussion anknüpfen, die einige Thesen Tocquevilles und Putnams kritisch oder zumindest differenziert sieht. So hat Roland Roth (2004) in seinem Aufsatz „Die dunkle Seite der Zivilgesellschaft“ bemerkt, dass „Zivilgesellschaft“ und „soziales Kapital“ (nach Putnams Verständnis) ,den Status eines Wundermittels erreicht [hätten] und „für fast alle gesellschaftlichen Probleme“ einsetzbar wären. (ebd.: 42f.) Roths Kritik richtet sich gegen die Annahme, dass Zivilgesellschaft automatisch „demokratieförderliche und demokratieverträgliche Wirkungen" entfaltet. Roth verteidigt seine Ansicht insbesondere gegen zwei zentrale Hypothesen der ,guten Zivilgesellschaft“, wie er es nennt: die Sozialisations- und die Transferhypothese. Diese formuliert er folgendermassen:

(1) Assoziationen sind für ihre Mitglieder Orte, an denen sie demokratische Tugenden erlernen und praktizieren können. (2) Diese zivilgesellschaftlichen Tugenden wirken sich positiv auf prosoziale Einstellungen (vor allem die Bereitschaft zu gegenseitiger Hilfe und Solidarität) aus und fördern politisches Vertrauen und demokratische Beteiligung. (ebd.: 45)

So gibt es in westlichen Demokratien, wie Roth darlegt, Assoziationen der sogenannten bad civil society. $\mathrm{Zu}$ diesen zählt er Gruppen und Zusammenschlüsse wie zum Beispiel rechtsextreme Gruppierungen oder islamistische Terrornetzwerke. Für ihre Mitglieder bieten solche Vereinigungen ,einige der Segnungen des ,sozialen Kapitals', denn sie schaffen möglicherweise Vertrauen, Geborgenheit und wechselseitige Unterstützung“. (ebd.: 45) Allerdings vermitteln sie ,zentrale demokratische Tugenden wie Zivilität im Sinne von Toleranz, Anerkennung Respekt und Gewaltfreiheit“ nicht. (ebd.: 45) Das Gegenteil ist vielmehr der Fall. So gehören Aus- 
schluss und Abwertung gegenüber anderen zu den zentralen, gruppenkonstituierenden Merkmalen. Roth spricht hier auch von „Brutstätten von antizivilen Stereotypen und Vorurteilen“. (ebd.: 45) Eine breite Vereinslandschaft ist per se kein Garant für eine demokratische Orientierung. Die deutsche Geschichte ist reich an Beispielen davon. „Das üppige Vereins- und Assoziationswesen der Kaiserzeit und der Weimarer Republik trug erheblich zur Stärkung antidemokratischer und abschottender Tendenzen. Die wilhelminische Vereinsmeierei reproduzierte in ihren geschlossenen Zirkeln nicht nur soziale Distanz, Standesdünkel und Reputationsgehabe, sondern prägte eine politikferne und herrschaftskonforme Untertanenmentalität“. (ebd.: 46) Einen weiteren Schwachpunkt stellt der Zugang zu Vereinen und Assoziationen dar. Mit Blick auf die Mitgliedschaft in rechtlich privilegierten Assoziationen und Vereinigungen, über die auch die Zugänge zu wichtigen Arbeitsmärkten und -netzwerken geregelt werden, fällt es schwer, nur von einer freiwilligen Mitgliedschaft zu sprechen. Zudem ist das Vereinswesen, so Roth, von sozialen Schichten und Milieus geprägt. Soziale Ungleichheit wird nicht automatisch durch ein Nebeneinander heterogener sozialer und kultureller Milieus abgebaut, sondern vielfach durch die Vereinsstrukturen produziert und reproduziert. So wird im ,gehobenen Bereich der Zivilgesellschaft“, wie Roth glaubhaft schildert, ,auf Exklusivität, soziale Distanz und Distinktion geachtet und werden diese durch Praktiken der sozialen Schliessung gesichert. Dies gilt nicht nur für Rotary Clubs; hierarchisch strukturiert sind auch die weniger prätentiösen Welten der Kultur-, Freizeit- und Sportvereine“. (ebd.: 48) Roth verweist hier auf den „Mittelschichtsbias“ zivilgesellschaftlichen Engagements, der nur eine von vielen Ausdrucksformen dieser Asymmetrie ist. (ebd.: 48)

\section{Wer engagiert sich in Vereinen der Schweiz?}

Allem Anschein nach steht die Bereitschaft zum freiwilligen Engagement in Zusammenhang mit soziodemografischen und sozioökonomischen Faktoren sowie Einstellungen und Werten. Dem Freiwilligen-Monitor der Schweiz 2016 ist zu entnehmen, dass formell freiwillig Tätige folgende charakteristische Eigenschaften aufweisen: Sie zeichnen sich durch einen hohen sozialen Status sowie einen fortgeschrittenen „Integrationsgrad hinsichtlich familiärer, freundschaftlicher oder beruflicher Beziehungen“ aus. (Freitag et al. 2016: 16) Wie in den vorherigen Studien ist ein formell freiwilliges Engagement bei Personen mit hoher Bildung, im mittleren Alterssegment (d. h. zwischen 40 und 64 Jahren), mit schulpflichtigen Kindern, in der deutschsprachigen Schweiz und auf dem Land gehäuft anzutreffen. Männer engagieren sich anteilig etwas häufiger formell freiwillig als Frauen (wobei der Unterschied statistisch nicht mehr signifikant ist); sie üben aber noch signifikant öfter als Frauen ein Ehrenamt aus. Freiwilliges Engagement wird, wie der Freiwilligen-Monitor zeigt, in hohem Masse von den individuellen Ressourcen beeinflusst. Dem sozialen Status kommt dabei eine zentrale Rolle zu. Höher gebildete und gutverdienende Personen üben häufiger ein freiwilliges Engagement aus, als Personen mit geringer Bildung und niedrigem Einkommen und sind zu höheren Anteilen ehrenamtlich tätig. Als wichtige Ursachen dafür gelten: Personen mit hoher Bildung verfügen über Kenntnisse und Fähigkeiten, die für ein freiwilliges Engagement unabdingbar sind. Personen mit einem hohen sozialen Status werden viel häufiger direkt durch Personen aus der Organisation 
oder Bekannte angesprochen und zum Engagement motiviert. (ebd.: 61) Ein weiterer wichtiger Aspekt ist, dass - wie schon in den vorherigen Befragungen - sich das „Engagement in Vereinen und Organisationen nach dem Rhythmus des Erwerbslebens richtet“. (ebd.: 59) Freiwillige Tätigkeiten in Vereinen werden in der Schweiz am häufigsten von den 40- bis unter 65-Jährigen (30\% sind formell freiwillig tätig und $14 \%$ ehrenamtlich engagiert) ausgeübt, also genau von den Menschen, die ,,am beruflichen Karrierehöhepunkt angelangt und meist gesellschaftlich wie familiär etabliert" sind. (ebd.: 61) Nach der Pensionierung, d. h. bei der Altersgruppe der 65- bis 79-Jährigen finden sich immer noch hohe Engagementquoten (26\% sind formell freiwillig tätig und $10 \%$ ehrenamtlich engagiert), die erst in den Altersgruppen der über 79-Jährigen deutlich absinken (nur noch $11 \%$ sind formell freiwillig oder $1 \%$ ehrenamtlich tätig). Für den Rückgang im höheren Alter sind vor allem körperliche und gesundheitliche Veränderungen verantwortlich. Jüngere Altersgruppen sind im Bereich des freiwilligen Engagements weniger vertreten. So zeigen beispielsweise junge Erwachsene (20 bis 39 Jahre) weniger freiwilliges Engagement (20\%) als Jugendliche der Altersgruppe von 15 bis 19 Jahren (22\%). Die geringere Quote des Engagements von jungen Erwachsenen im Vergleich zu Jugendlichen lässt sich der Häufung der Belastungen (im Zusammenhang mit beruflichen Aus-/Weiterbildungen und familiären Verpflichtungen) zuordnen. (ebd.: 59f.) Ein ähnliches Muster zeigt sich auch beim Ehrenamt in Vereinen: Am stärksten sind die Personen der Altersgruppe von 40 bis 64 Jahren ehrenamtlich engagiert. Die Erklärung dafür liegt, so vermuten Freitag et al., wahrscheinlich darin, dass ein ,fortgeschrittenes Alter und entsprechende Lebenserfahrung gepaart mit der beruflichen Etablierung und dem damit verbundenen Prestige“ ein Hauptgrund für die Übernahme solcher Tätigkeiten zu sein scheint. (ebd.: 60)

Laut Angaben des Freiwilligen-Monitors Schweiz 2016 engagieren sich Personen, die mit schulpflichtigen Kindern im gleichen Haushalt leben, anteilig häufiger als der Durchschnitt der Bevölkerung. Über die Schule und die Freizeitaktivitäten der Kinder erschliessen sich ihnen neue Kontakte und Netzwerke, die das Ausüben von formell freiwilligen Tätigkeiten erleichtern. (ebd.: 66ff.) Die zeitliche Verfügbarkeit bedeutet grundsätzlich keine Einschränkung für die Aufnahme einer formell freiwilligen Tätigkeit. Personen, die über genügend zeitliche Ressourcen für ein freiwilliges Engagement in Vereinen und Organisationen verfügen, wie etwa Rentnerinnen und Rentner, Arbeitslose oder Teilzeit-Erwerbstätige, verzeichnen einen signifikant geringeren Anteil formell freiwillig Tätiger an der Wohnbevölkerung über 15 Jahre als Vollzeit-Erwerbstätige. (ebd.: 62ff.)

Auffallend sind die früher schon erwähnten regionalen Unterschiede im freiwilligen Engagement in der Schweiz. So ist der Anteil formell Freiwilliger in der deutschsprachigen Schweiz deutlich höher als in der Suisse romande und in der italienischsprachigen Schweiz. (ebd.: 70ff.) In allen Regionen lässt sich zudem ein Unterschied zwischen Städten, Agglomerationen und ländlichen Gemeinden feststellen. Bewohnerinnen und Bewohner ländlicher Gemeinden sind signifikant häufiger formell freiwillig tätig (32\%) als Bewohnerinnen und Bewohner von Agglomerationen (23\%) und diese wiederum häufiger als Bewohnerinnen und Bewohner von Städten (21\%). Zudem engagieren sich Bewohnerinnen und Bewohner auf dem Land deutlich häufiger ehrenamtlich (13\%) als Personen aus Agglomerationen (9\%) oder urbanen Gebieten (8\%). (ebd.: 69) Erklärt wird die auffallend höhere Engagementsquote im ländlichen Raum mit 
der , geringere[n] Anonymität und d[em] damit verbundene[n] Moment der sozialen Wünschbarkeit ebenso wie gesellschaftliche[n] Sanktionsmöglichkeiten der Nichtbeteiligung“. Informelle soziale Kontrolle sei, wie Freitag et al. betonen, in einer städtischen Umgebung viel schwächer. (ebd.: 69)

Abschliessend möchte ich noch anmerken, dass in der Schweiz freiwilliges Engagement in Vereinen und Organisationen hauptsächlich auf lokaler Ebene stattfindet. Über 90 Prozent aller formell freiwillig Tätigen sind in lokalen Vereinen engagiert, zum Beispiel in der Nachbarschaft, im Dorf oder städtischen Quartier, oder in der Region. Nur wenige üben eine Tätigkeit auf gesamtschweizerischer oder sogar globaler Ebene aus. (ebd.: 55)

\subsection{Freiwillige Engagement von (neuen) Zugewanderten in der Schweiz}

In diesem Kapitel werde ich mich dem Forschungsstand zum freiwilligen Engagement von Ausländerinnen und Ausländern in der Schweiz widmen. Eine detaillierte Analyse zum Ausmass, zu den Bereichen, den Inhalten und zur Ausrichtung des freiwilligen Engagements von Personen mit Migrationshintergrund liefert der jüngste Freiwilligen-Monitor mit seiner „Ausländerstichprobe“. (Freitag et al. 2016: 183ff.) Laut den Zahlen des Freiwilligen-Monitors 2016 engagieren sich 14 Prozent der in der Schweiz lebenden Ausländerinnen und Ausländer in einem Verein oder in einer Organisation freiwillig. (ebd.: 187) Menschen mit Migrationshintergrund sind somit deutlich weniger freiwillig tätig als Schweizerinnen und Schweizer (35\%). Ein ähnliches Muster gilt gleichwohl für die ehrenamtliche Tätigkeit: „Der Anteil der Ausländerinnen und Ausländer mit ehrenamtlichen Engagement an den formell Freiwilligen liegt bei rund 25 Prozent und fällt damit überzufällig kleiner aus als das ehrenamtliche Engagement der formell freiwillig tätigen Schweizerinnen und Schweizer (rund 39 Prozent)“. (ebd.: 189) Dieser Umstand kann, wie Freitag et al. betonen, auf mangelnde Sprachkenntnis, fehlende Verwurzelung am neuen Heimatort oder wenig ausgeprägte soziale Netzwerke zurückgeführt werden. (ebd.: 20) Betrachtet man das Bild etwas differenzierter, zeigt sich für die ausländische Bevölkerung, dass sich diese in einzelnen Bereichen und Formen der Freiwilligkeit nicht von Schweizerinnen und Schweizern unterscheiden. Freitag et al. heben sogar hervor, dass ihr Engagementverhalten ,häufig auch Ähnlichkeiten mit dem Verhalten der gebürtigen Schweizerinnen und Schweizer" aufweist. (ebd.: 19f.) Es gibt zum Beispiel Bereiche des formellen freiwilligen Engagements, in denen ausländische Personen ähnliche Engagementquoten wie Schweizerinnen und Schweizer aufweisen. So engagieren sich die meisten Ausländerinnen und Ausländer, wie die meisten Schweizerinnen und Schweizer, in Sportvereinen. Am zweithäufigsten sind sie in kirchlichen Organisationen freiwillig tätig, gefolgt vom Engagement in kulturellen und sozialen, karitativen oder gemeinnützigen Organisationen. Eine besondere Stellung nehmen bei Ausländerinnen und Ausländern die Migrantenvereine ein. Gebürtige Schweizerinnen und Schweizer sind in diesen kaum aktiv, sondern ,,tendenziell [stärker] in karitativen Organisationen, in Hobbyvereinen, Jugendorganisationen und (natürlich) im politischen Bereich eingebunden“. (ebd.: 193) Freitag et al. weisen ausdrücklich drauf hin, dass die in ihrer Analyse ermittelten Unterschiede nach Engagementbereichen statistisch rein zufällig sind. So kommen sie zum Fazit, dass sowohl „Personen anderer Nationalität als auch Gebürtige (...) in ähnlichen 
Vereins- und Organisationsstrukturen freiwillig tätig sind“. (ebd.: 193) Ähnliches lässt sich auch in Bezug auf Umfang und Motive des Engagements sowie auf den schon erörterten Einfluss des Alters und des sozioökonomischen Status auf die Übernahme einer freiwilligen Tätigkeit beobachten.

Das Engagement von Ausländerinnen und Ausländern ist wie das von Schweizerinnen und Schweizern lokal beschränkt. 86 Prozent der Ausländerinnen und Ausländer sowie der eingebürgerten Schweizerinnen und Schweizern engagieren sich im unmittelbaren lokalen Lebensumfeld, d. h. in der Nachbarschaft, im Dorf oder in der Region. Ihre Engagementquote liegt damit nur geringfügig unter dem der gebürtigen Schweizerinnen und Schweizer (94\%). Der Grund für diesen Unterschied liegt offenbar darin, dass Schweizerinnen und Schweizer stärker lokal verankert sind als Personen mit Migrationshintergrund. (Freitag et al. 2016: 194f.) Beim Engagement auf globaler Ebene ist dagegen der Anteil der ausländischen Personen (9\%) höher als der der Schweizerinnen und Schweizer (2\%). Aufgrund der geringen Fallzahlen ist der Unterschied aber nicht statistisch signifikant und mit einer relativ hohen Unsicherheit behaftet. (ebd.: 194f.)

Lediglich im Bereich der öffentlichen Massnahmen zur Steigerung des freiwilligen Engagements ist ein signifikanter Unterschied zwischen der ausländischen und der Schweizer Bevölkerung zu erkennen: Mehr als die Gebürtigen ist ungefähr die Hälfte aller Personen mit anderer Staatsangehörigkeit der Meinung, dass es eine bessere Information über Gelegenheiten zu ehrenamtlichen und freiwilligen Tätigkeiten braucht. Dies zeigt deutlich, wie Freitag et al. explizit betonen, dass ,in Bezug auf die Informationslage für Ausländerinnen und Ausländer Nachholbedarf seitens der Öffentlichkeit“ besteht. (ebd.: 20) Zudem ist es auffallend, dass die eingebürgerten Personen ,im Vergleich zu den gebürtigen Schweizerinnen und Schweizern von den Vereinen eine höhere Anerkennung, mehr fachliche Unterstützung und eine stärkere Begrenzung der Tätigkeit wünschen“. (ebd.: 20)

Im Folgenden möchte ich einige methodenkritische Überlegungen zur Repräsentativität und Zusammensetzung der Ausländerstichprobe des Freiwilligen-Monitors anstellen. Diese sind meiner Meinung nach bei der Interpretation der ermittelten Ergebnisse gebührend zu berücksichtigen. So sind erstens die Ausländerinnen und Ausländer in der Stichprobe überrepräsentiert. Der Anteil der Ausländerinnen und Ausländer mit 33 Prozent in der Stichprobe weicht um neun Prozentpunkte vom Anteil in der Gesamtbevölkerung (24 \%) ab. Eine ähnliche Situation ergibt sich für bestimmte wichtige Herkunftsländer wie Deutschland, Italien, Portugal, Frankreich und Serbien. (ebd.: 185) Zweitens führt das Auswahlverfahren zu einer Überrepräsentanz gut integrierter Ausländerinnen und Ausländer, da andere Gruppen von Migrantinnen und Migranten aufgrund fehlender Kenntnisse einer der schweizerischen Amtssprachen (deutsch, französisch oder italienisch) ausgeschlossen sind. Daher ist eine Verzerrung der Stichprobenzusammensetzung zu erwarten. Freitag und Mitarbeitende sind sich jedoch bewusst, dass die Ausländerinnen und Ausländer, die in die Stichprobe gelangt sind, ,bereits zu den sozial besser gestellten und gut integrierten Nationalitäten in der Schweiz gehören. Zudem bekunden diese ausländischen Befragten naturgemäss weniger Mühe, gesellschaftliche und kulturelle Integrationshürden in der Schweiz zu überwinden“. (Freitag et al. 2016: 186). 
Die Ausländerstichprobe des Schweizer Freiwilligen-Monitors, und das ist mir wichtig zu erwähnen, enthält eine Vielzahl von informativen quantitativen Daten zum Engagementverhalten der ausländischen Wohnbevölkerung in der Schweiz. Allerdings sind aufgrund geringer Fallzahlen kaum klare Muster oder Tendenzen hinsichtlich der Herkunftsländer erkennbar. So sind Rückschlüsse auf das Engagementverhalten von Expatriates in der Schweiz nicht möglich, da diese zu heterogen sind. Sie unterscheiden sich zum Beispiel nach Sprache, Aufenthaltsdauer, nationaler/kultureller Zugehörigkeit, Karriereplänen und Lebensumständen (vgl. Kap. 2 und 3 meiner Arbeit). Schwachpunkt dieser Ausländerstichprobe ist, dass die Ausländerinnen und Ausländer nur nach einigen wenigen Kategorien wie sozialer Status, Bildung oder Herkunftsland klassifiziert werden. Das erschwert eine differenzierte Analyse, die besondere Merkmale und Eigenschaften der Expatriate-Bevölkerung berücksichtigt, erheblich. Am Freiwilligen-Monitor haben vermutlich auch Expatriates teilgenommen, doch wahrscheinlich nur in geringer Anzahl, da viele unter ihnen aufgrund mangelnder Kenntnisse einer der drei Landessprachen ausgeschlossen waren. Im Unterschied dazu habe ich in meine Studie Expatriates eingeschlossen, die (mit wenigen Ausnahmen) keiner der landesüblichen Sprachen mächtig sind.

In meiner eigenen Untersuchung zu Expatriates und deren Engagement habe ich mich sehr stark an Sandro Cattacin und Dagmar Domenig ausgerichtet, die sich in ihrer Studie „Inseln transnationaler Mobilität. Freiwilliges Engagement in Vereinen mobiler Menschen in der Schweiz“ (2012) mit einer ähnlichen Fragestellung beschäftigen. So legen sie in ihrer Arbeit empirisch den Fokus auf ,diejenigen Motivationen freiwilligen Engagements von Personen in Vereinen (...) die sich auf deren Mobilitätshintergrund beziehen“. (Cattacin/Domenig 2012: 17)

In ihrer Einleitung fassen Cattacin und Domenig die Ausgangslage hinsichtlich des freiwilligen Engagements mobiler Menschen in der Schweiz zusammen. Diese kann als qualitatives Gegenstück zur quantitativ orientierten Ausländerstichprobe des Freiwilligen-Monitors verstanden werden:

Das freiwillige Engagement transnational mobiler Menschen in kollektiven Organisationsformen ist durchaus vergleichbar mit demjenigen der Einheimischen, nur sind mobile Menschen mit einem ausländischen Pass im Schnitt in der Schweiz zehn Prozent weniger freiwillig tätig, was durchaus auch für andere Länder zutrifft. Daraus einfach abzuleiten, mobile Menschen seien an freiwilliger Arbeit weniger interessiert, wäre jedoch falsch. Es könnten zwar Hypothesen formuliert werden, die dieses statistische Resultat damit erklären, dass es eher schwierig ist, Freiwillige zu finden, die auch bereit sind, sich dem spezifischen Gemisch von sozialer Kontrolle und Verantwortung in einem auf mobilen Personen basierten Verein auszusetzen oder sich den Luxus, für andere da zu sein, überhaupt leisten zu können. Denn die meisten Personen mit Migrationshintergrund sind ja nach dem Umzug zuerst einmal mit sich und ihrer Familie beschäftigt, und zwar sowohl mir ihrer eigenen als auch mit den familiären Netzwerken am Ursprungsort. Dies könnte zur Folge haben, dass der soziale Druck, sich auch im Zielland zu engagieren, relativ gering wäre. Zudem wissen viele mobile Menschen ja meist nicht, wie lange sie bleiben. (ebd.: 15f.)

In ihrer Studie fragen Cattacin und Domenig nach den Motiven, die transnational mobile Menschen zu einem Engagement in einem Verein bewegen. Sie haben im urbanen Kontext (Zürich, Lausanne und Genf) mit Vereinsmitgliedern und Präsidentinnen und Präsidenten der Vereine mobiler Menschen Gespräche geführt. Aus ihrer Analyse geht hervor, dass sich die Aufgabenbereiche und Funktionen der Vereine entsprechend den unterschiedlichen geschichtlichen Herausforderungen durch die internationale Mobilität verändern. Die Tätigkeitsfelder variieren je nach politisch-ökonomischen Rahmenbedingungen. Ausgehend davon identifizieren Cattacin 
und Domenig vier verschiedene Konstellationen: (1) frühere, traditionelle Migration im Fordismus, (2) neue reguläre Mobilität im Flexibilismus, (3) neue irreguläre Mobilität im Flexibilismus sowie (4) politische Asylmigration. (ebd.: 17f.) Während der früheren Migration im Fordismus sind hauptsächlich Personen in Vereinen tätig, die im Zuge des wirtschaftlichen Aufschwungs nach dem Zweiten Weltkrieg in die Schweiz gekommen sind; dabei handelt es sich vorwiegend um Vereine von Menschen aus Italien und Spanien. Bei der neueren regulären Mobilität schliessen sich in Vereinen vor allem Menschen zusammen, „die zur transnationalen mobilen internationalen Elite gehören und sich organisieren; dazu gehören zum Beispiel Vereine von Menschen aus den USA oder Schweden“ (ebd.: 18), d. h. also auch Vereine und Organisationen von Expatriates in der Schweiz. In der Zeit der neueren irregulären Mobilität organisieren sich in Vereinen mobile Menschen, die oft nicht hochqualifiziert sind (manchmal aber schon) und meist unter prekären Aufenthaltsbedingungen in der Schweiz leben. Als Beispiel erwähnen Cattacin und Domenig Personen aus Südamerika. In der andauernden Periode der politischen Asylmigration engagieren sich in Vereinen Personen, die in die Schweiz geflohen sind und sich dort oft zu einer Diaspora vereinigen. Viele dieser Menschen kommen aus dem Kosovo und Afrika. (ebd.: 18)

Cattacin und Domenig betonen in ihrer Einleitung zum mobilitätsbasierten Vereinswesen, dass Vereine nicht nur einen individuellen und gesellschaftlichen Mehrwert bringen, sondern auch das potenzielle Risiko von sozialer Ausgrenzung bergen, die zu einer unerwünschten Ghettobildung führen könnte. Von daher ist es für sie nicht verwunderlich, dass die ,gemeinschaftliche Vereinigung von mobilen Personen (...) in der wissenschaftlichen Literatur nicht nur multidimensional, sondern in ihrer Konsequenz für die Gesellschaft auch ambivalent beurteilt" (ebd.: 22) wird. Die angesprochene Ambivalenz des Vereinswesens fassen Cattacin und Domenig in der folgenden Tabelle kurz zusammen:

Tabelle 3: Ambivalenz freiwilliger Vereinigungen mobiler Menschen

\begin{tabular}{|l|l|l|}
\hline Auswirkungsbereiche & $\begin{array}{l}\text { Günstige Faktoren für die gesell- } \\
\text { schaftliche Reproduktion }\end{array}$ & $\begin{array}{l}\text { Ungünstige Faktoren für die gesell- } \\
\text { schaftliche Reproduktion }\end{array}$ \\
\hline Politische Auswirkungen & $\begin{array}{l}\text { Vereine als Orte zivilgesellschaftli- } \\
\text { cher Kontrolle über die Regie- } \\
\text { rungstätigkeit (governance) }\end{array}$ & $\begin{array}{l}\text { Vereine als Orte des Klientelismus } \\
\text { und als Alibiinstanz sektorieller Poli- } \\
\text { tik }\end{array}$ \\
\hline Ökonomische Auswirkungen & $\begin{array}{l}\text { Vereine als Basis einer auf Konkur- } \\
\text { renz basierenden Marktwirtschaft }\end{array}$ & $\begin{array}{l}\text { Vereine als Orte der Innovationsblo- } \\
\text { ckierung durch soziale Kontrolle }\end{array}$ \\
\hline Auswirkungen auf die Identität & $\begin{array}{l}\text { Vereine als Orte der Stabilisierung } \\
\text { der Identität }\end{array}$ & Vereine als Ghettos \\
\hline
\end{tabular}

Quelle: Cattacin/Domenig 2012: 22.

Auf Basis ihrer empirischen Befunde beschreiben Cattacin und Domenig grundsätzlich - und das unabhängig vom Vereinstypus - drei Motivgruppen, die für ein Vereinsengagement mobiler Menschen Bedeutung haben: objektive, subjektive und soziale Motive. Diese unterscheiden 
sich vor allem individuell, zum Beispiel nach der Dauer des Aufenthaltes im Zielland Schweiz und der Verfügbarkeit sozialen Kapitals bei der Einreise. (ebd.: 125)

Nach ihrer Ankunft im Zielland suchen Zuwandererinnen und Zuwanderer Vereine vor allem aus strategischen oder objektiven Motiven auf. Ihr ,Engagement [lässt sich von] Nützlichkeitsgründen [leiten] “. (ebd.: 122) Für sie ist Sicherung der Existenz ein Leitmotiv ihres Engagements. Zu diesem Zweck visieren sie ,materielle Chancen an und [nehmen] bestehende Rechte in Anspruch“ (ebd.: 122). Daher begreifen Cattacin und Domenig Vereine als Orte,

an denen diese Ziele verfolgt werden können, indem hier wichtige Informationen ausgetauscht werden, beispielsweise über offenen Arbeitsstellen, gute Ärztinnen und Ärzte, preiswerte Autowerkstätten oder auch einfach nur über das Ausfüllen von Steuerformularen. Sie erweitern und stabilisieren soziales Kapital, erhöhen Wissen, bieten Sprachkurse an und führen Veranstaltungen zu Fragen der Gesundheit oder der Schule durch oder wenden sich an die Öffentlichkeit, um Diskriminierungen und Rassismus anzuprangern. Auch sind Vereine Orte alternativer Karrieren oder auch Zwischenstationen einer unterbrochenen Karriere (vor allem bei nicht berufstätigen Frauen von Expats). (ebd.: 122, Herv. i. O.)

Vereine sind gerade zum Zeitpunkt der Ankunft wichtig. Sie können für Neuankömmlinge eine wichtige Unterstützung beim Aufbau und bei der Stärkung der Beziehungen zwischen Eingewanderten und Einheimischen darstellen. (ebd.: 122f.)

In einem späteren Stadium, wenn sich die meisten Befürchtungen der Mobilen vor existenziellen Herausforderungen verflüchtigt haben, stehen identitäre Fragen im Vordergrund. Es sind nun vorwiegend subjektive Gründe, die dafür sorgen, dass sich Migrierte in Vereinen engagieren. Der Wunsch nach Würde ist ein Antrieb, sich einzusetzen. Denn in einem Verein lernen mobile Menschen, wie Cattacin und Domenig klar erkennen, für sich selbst zu sorgen und ,sich selbst zu sein“. (ebd.: 123) Vereine wirken deswegen

\footnotetext{
identitätsstabilisierend, indem sie als Rückzugsräume und gewissermassen als Orte der identitären Selbstvergewisserung dienen. Manchmal werden die Vereine auch Refugien für die Reflexion des Leidens an der Nichtanerkennung oder für die Suche nach Würde, die dort ganz einfach durch Sinnstiftung erfahren werden kann. Auf diese Weise werden Vereine auch zu Orten der Umkehrung von Stigmatisierung (Goffman 1963), indem Fremdheitserfahrungen und Entfremdungen nicht einfach nur verständlich gemacht, sondern über die Gruppe normalisiert und deindividualisiert werden. Gründe des Unwohlseins werden dabei nicht einfach sich selbst, sondern anderen zugeordnet. Und so kann man im Verein wieder stolz auf seinen Ursprung und seine Sprache sein und erhält erst noch Kraft, die letztlich nicht nur den Einzelnen, sondern das ganze Kollektiv stärkt. (ebd.: 123f.)
}

Vereine sind für mobile Menschen als „Rückzugsinseln“ für allerlei besonders wertvoll. (ebd.: 124) Sie helfen ihnen dabei, ihr individuelles Wohlbefinden zu steigern. Vereine verstehen sich als geschützte Räume, in denen mobile Menschen Vertrauen und Zuversicht aufbauen, was wiederum als eine wichtige Ressource im Zusammenleben sowie bei der Konstitution von Gesellschaft erlebt wird. (ebd.: 124) Vereine bilden, wie Cattacin und Domenig überzeugend zeigen, ,zentrale Stützen im Mobilitätsprozess, denn eine durch das Vereinsleben gestärkte Persönlichkeit kann sich auch ausserhalb des Vereins selbstbewusster durchsetzen und kreativer mit Vielfalt und Ambivalenz umgehen“. (ebd.: 124)

Mobile Menschen engagieren sich auch aus sozialen und parapolitischen Motiven. Der Anschluss an einen Verein oder an die Zivilgesellschaft ergibt sich aus dem Leitmotiv der Soziabilität heraus. (ebd.: 124) Der Wunsch nach Soziabilität ist allerdings ein Bedürfnis, das bei 
Mobilen erst später hinzukommt, wenn sie eine gewisse stabile Identität aufgebaut haben. Es geht dabei hauptsächlich um Fragen sozialer Eingebundenheit sowohl im Zielland als auch im Herkunftsland. (ebd.: 125) Anlass hierfür ist primär die ,identitäre Vermittlung des Besonderen gegenüber den eigenen Kindern“, die den Ursprungsort vielfach nur aus Erzählungen oder Ferienerlebnissen kennen. (ebd.: 125)

Soziabilität ist also für mobile Menschen wichtig, denn sie ermöglicht es ihnen, mit Anderen, mit denen sie sich verbunden fühlen, Dinge gemeinsam zu tun. Vereine bieten durch das gemeinsame Zelebrieren von Gemeinsamkeiten verbindende Orientierungen, die nach innen als gruppentypische Besonderheiten erlebt werden. Die Definition ihrer Eigenheiten erlaubt es der Gruppe, nach aussen zu treten und als solche in der Öffentlichkeit wahrgenommen zu werden, wie etwa im Fall der Zusammenarbeit mit lokalen Behörden oder Schulen. (ebd.: 124)

Die nachfolgende Tabelle fasst die gewonnenen Erkenntnisse noch einmal zusammen.

Tabelle 4: Vereinsengagement - Gemeinsamkeiten in den Motiven mobiler Menschen

\begin{tabular}{|c|c|c|}
\hline Leitmotiv & Innendynamik & Aussendynamik \\
\hline \multicolumn{3}{|c|}{ Leitmotiv: Existenz oder materielle Chancen packen und Rechte beanspruchen } \\
\hline Objektive Gründe & $\begin{array}{l}\text { Suche nach Inklusionshilfen in Sys- } \\
\text { temen oder nach alternativen para- } \\
\text { professionellen Erfahrungen }\end{array}$ & $\begin{array}{l}\text { Suche nach indirekten, über den Ver- } \\
\text { ein gesteuerten Zugängen zu politi- } \\
\text { schen Einflussmöglichkeiten und zu } \\
\text { Nutzen bringenden Netzwerken }\end{array}$ \\
\hline \multicolumn{3}{|c|}{ Leitmotiv: Würde oder sich um sich selber sorgen und lernen, sich selbst zu sein } \\
\hline Subjektive Gründe & $\begin{array}{l}\text { Suche nach identitärer Stabilisie- } \\
\text { rung }\end{array}$ & $\begin{array}{l}\text { Suche nach Stärkung identitärer } \\
\text { Selbstbezüge im Alltag }\end{array}$ \\
\hline \multicolumn{3}{|c|}{ Leitmotiv: Soziabilität oder Gemeinsamkeiten zelebrieren und soziale Anerkennung einfordern } \\
\hline Soziale Gründe & $\begin{array}{l}\text { Suche und Erarbeitung von legiti- } \\
\text { men Identitätsdefinitionen, die mit } \\
\text { dem Ursprungs- und Zielland kom- } \\
\text { patibel sind }\end{array}$ & $\begin{array}{l}\text { Suche nach Stärkung (durch den Be- } \\
\text { zug auf den Verein) von identitären } \\
\text { Selbstbezügen in Aushandlungspro- } \\
\text { zessen }\end{array}$ \\
\hline
\end{tabular}

Quelle: Eigene Darstellung in Anlehnung an Cattacin/Domenig 2012: 123.

Cattacin und Domenig halten als Ergebnis Folgendes fest:

In der Regel sind Vereine transnational mobiler Menschen Orte der gesellschaftlichen Öffnung. Orte, die sich durch intensive Innen- und Aussenbeziehungen auszeichnen, die (...) Vorurteile abbauen und den Respekt vor Verschiedenheit fördern (...). Als zentrale Informationsdrehscheibe zwischen den mobilen Menschen und der lokalen Bevölkerung arbeiten Vereine an gesellschaftlichen Schnittstellen und Übergängen und leisten dadurch einen wesentlichen, wenn nicht gar den wichtigsten Beitrag zur sozialen und systemischen Inklusion mobiler Menschen (...). Darüber hinaus sind es primär die Vereine, die sich um diejenigen kümmern, deren Mobilitätsprojekt scheitert, und die durch die Exklusion entstehenden Probleme absorbieren beziehungsweise auffangen. (ebd.: 125)

Die Mitgliedschaft und das Engagement in Vereinen können, wie früher schon ausgeführt, auch ambivalente Wirkungen haben, wenn es sich zum Beispiel um solche Vereine handelt, die die 
Entstehung von Subkulturen fördern und Abschottungstendenzen verstärken. Cattacin und Domenig greifen diesen Diskussionsfaden auf und führen ihn weiter, indem sie das Verhältnis von Mehrheit und Minderheit in der Gesellschaft thematisieren:

Vereine mobiler Menschen können aber auch - ähnlich wie Kirchen und Lifestyle-Gruppen - zu nahezu abgeschlossenen Welten werden, wobei dies eher die Ausnahme ist. Solche Abschottungen sagen dabei meist mehr über die Gesellschaft aus, in der sie stattfinden, als über die Menschen, die sich dahinter verbergen. Denn Ghettobildungen sind Ausdruck von Abgrenzung der Mehrheit gegenüber der Minderheit und somit Produkte symbolischer oder physischer Gewalt. Erst die gesellschaftliche Inklusion der Minderheit durch die Mehrheit kann solche Ghettos aufbrechen und die Minderheit am gesellschaftlichen Diskurs gleichberechtigt und gleichwertig beteiligen. (ebd.: 126)

Sandro Cattacin und Dagmar Domenig haben eine höchst informative und sehr interessante Studie zum freiwilligen Engagement transnational mobiler Menschen vorgelegt, die einen für mein Forschungsanliegen geeigneten Zugang darstellt. Besonders wertvoll scheint mir ihre Betonung der Notwendigkeit, qualitative Methoden einzusetzen und die transnationale Forschungsperspektive auf das empirische Beispiel mobiler Menschen anzuwenden. Die Kombination beider Herangehensweisen ist meiner Meinung zwingend erforderlich, um sich der Frage nach dem Engagement von Expatriates zu nähern (vgl. hierzu Kap. 2, 3 und 4 meiner Arbeit). Cattacin und Domenig haben mir wichtige Impulse für das Verständnis der transnationalen Perspektive gegeben. Ihr Fazit dazu fällt sehr eindeutig aus:

\footnotetext{
Transnationale Mobilität wirkt auf Menschen auf verschiedene Weise, indem diese kurzfristig zu einem Abbau sozialer Beziehungen, längerfristig jedoch zu einem Aufbau neuer Beziehungen führt, die an Komplexität gewinnen, da sie neu überterritorial entstehen. Die Intensität der Beziehungen zum Ursprungsort - oder bei nomadischer Mobilität zu den früheren Lebensorten - nimmt zwar ab, doch bleiben die Beziehungen im Wesentlichen bestehen und werden durch neue Beziehungen ständig ergänzt (...). Insgesamt kommt es deshalb nicht zu einer Abnahme der persönlichen Beziehungen, sondern zum Aufbau eines weit komplexeren, transnationalen und lokalen Beziehungsgeflechts. (ebd.: 126)
}

Wie ich später in meinem empirischen Teil darlegen werde, beteiligt sich ein Grossteil der von mir erforschten Expatriates in Vereinen, die explizit auf ihre Bedürfnisse und Wünsche ausgerichtet sind.

Eine speziell auf Expatriates fokussierte Studie gibt es in der Schweiz für die Region Basel. (vgl. Wiener/Grossmann 2011) Ihr erklärtes Ziel ist es, „die Lebenswelt und Interessen sowie die Potenziale der Expats in der Region Basel systematisch zu erfassen“. (ebd.: 5) Aufbauend auf einer Stakeholderanalyse mit Interviews, deskriptiven Statistiken und Fokusgruppen nähert sich die Studie dem Erkenntnisobjekt Expatriates im Raum Basel aus unterschiedlichen Perspektiven. (ebd.: 21f.) Trotz grossen Anstrengungen gelingt es Daniel Wiener und Marco Grossmann nicht, detaillierte Daten zu wichtigen Merkmalen der Expatriate-Bevölkerung in Basel zu erhalten. So verwenden sie stattdessen aktuelle gesamtschweizerische Daten zu bestimmten Aspekten (wie etwa Anteile der Ausländerinnen und Ausländer an der Gesamtbevölkerung, Herkunft der Einwanderinnen und Einwanderer, Bildung und Einkommen nach Nationalitäten). Dabei merken sie an, dass es sich nur um Näherungswerte handelt, diese Werte nur Tendenzen und erste Hinweise liefern: „Aufgrund der nur vagen statistischen Definition von Expats und dem Mangel an vorhandenen Daten lassen sich keine bestimmten Aussagen über 
,die Expats in Basel' machen. An dieser Stelle können nur Einschätzungen vorgenommen werden“. (ebd.: 41) Im Anschluss an die sekundärstatistische Analyse untersuchen Wiener und Grossmann die Potenziale und Hindernisse einer gesellschaftlichen Integration von Expatriates im Raum Basel. So zeigen sie Potenziale auf mehreren Ebenen (der gesellschaftlichen, volkswirtschaftlichen, betriebswirtschaftlichen) auf. Eine besondere Wichtigkeit komme, so Wiener und Grossmann, ,der Integration der Expats in soziale und/oder gesellschaftliche Fragen und Engagements“ $\mathrm{zu}$, denn dies könne ,einen echten Mehrwert generieren und beispielsweise Vereinen helfen, ihren Mitgliederschwund einzudämmen.“. (ebd.: 44)

Doch werden in der Studie auch einige Hindernisse auf dem Gebiet der freiwilligen Arbeit genannt. Aus den qualitativen Interviews geht hervor, dass insgesamt sechs Hindernisse „die Integration von Expats erschweren“. (ebd.: 44) Es sind: Integration nur innerhalb des Unternehmens, Parallelgesellschaften, Sprachbarrieren, Bildung der Kinder/Jugendlicher, schwerverständliche (soziale) Regeln, Fremdenfeindlichkeit. (ebd.: 45ff.)

Die Kategorie „Integration nur innerhalb des Unternehmens“ fördert eine Art „Campus-Mentalität", die zum einen ein starkes Integrationshindernis darstellt, zum andern eine Erweiterung des Beziehungsnetzes bedeutet, da auf dem Campus neben Expatriates auch Einheimische arbeiten. (ebd.: 45)

Nach Wiener und Grossmann können Parallelgesellschaften ein tatsächliches Problem sein. Ein Integrationshemmnis stellen sie allerdings nur dann dar, wenn ,zu wenig Austauschmöglichkeiten und Berührungspunkte mit der Gesellschaft ausserhalb dieser Gruppe vorhanden [sind]“. (ebd.: 46) Oft sind sie lediglich die Folge anderer Hindernisse wie der Sprache oder der getrennten Lernangebote für den Expatriate-Nachwuchs. Eine besondere Problematik bei Parallelgesellschaften ergibt sich, wie Wiener und Grossmann darlegen, dann, ,wenn es zu einer ,Gettoisierung' kommt, respektive der nicht arbeitstätige Ehepartner überhaupt keine Berührungspunkte zur weiteren Umwelt pflegt“". (ebd.: 46)

Sprachbarrieren erweisen sich nach der Meinung aller interviewten Stakeholder als eine hohe Hürde für die Expatriates. Insbesondere das in Basel gesprochene Schweizerdeutsch erscheint vielen als grosse Herausforderung, vor allem dann, wenn sie die erworbenen Deutschkenntnisse auf Grund des Dialekts nur beschränkt nutzen können. Uneinig sind sich die interviewten Personen, ob dies heisst, dass die Expatriates besser Deutsch lernen müssen, oder Basel mehr Informationen auf Englisch und anderen Sprachen anbieten soll. „Prinzipiell scheint der Druck“, so die allgemeine Einschätzung, „Deutsch lernen zu müssen, speziell im Kanton Basel-Stadt gering zu sein“. (ebd.: 46)

Was die Bildung der Kinder/Jugendlicher in Basel angeht, haben die Interviewten unterschiedliche Auffassungen. Sie nennen zum Beispiel die Vor- und Nachteile der International School. Insbesondere ist ihnen bewusst, dass diese eine wichtige Rolle bei der Integration von Expatriates und ihrem Nachwuchs spielen. Wiener und Grossman schliessen sich dieser Einschätzung an und konstatieren:

Die International School hat so den Vorteil, dass ihre Abschlüsse international anerkannt sind. Jedoch wird sie oft verschlossen und elitär und daher als Integrationshemmnis gesehen. Auf der anderen Seite sind staatliche Schulen ein guter Integrator, sowohl für die Kinder, wie auch die Eltern, haben allerdings unter Umständen 
das Problem der internationalen Anerkennung. Während die International School besonders für Expats interessant ist, die nur sehr kurz in der Schweiz sind, sollten die staatlichen Angebote vor allem von Expats genutzt werden, die sich länger in Basel aufhalten. (ebd.: 47f.)

Für viele Expatriates sind manche sozialen Regeln im Zusammenleben schwerverständlich. Denn ,einerseits sind [diese] nicht alle nachvollziehbar. Andererseits sind [sie] oft nicht bekannt oder unverständlich, da in deutscher Sprache verfasst“. (ebd.: 47) Hinzu kommt, dass diese Regeln ein grosses Hindernis „,besonders bei Fragen rund um Versicherungen, Hausregeln und im Kontakt mit den Behörden" sind.

Genauso haben die Interviewten eine unterschiedliche Meinung zum Thema Fremdenfeindlichkeit. Menschen mit ausländischen Wurzeln und Menschen mit Schweizer Herkunft unterscheiden sich in ihrer Wahrnehmung. Während die Personen Schweizer Abstammung die Region Basel als sehr weltoffen wahrnehmen, erleben die Personen mit Migrationshintergrund eher ablehnende bis fremdenfeindliche Gefühle ihnen gegenüber. Diese Einschätzung leiten sie unter anderem aus ihren persönlichen Erfahrungen ab, ,beispielsweise während der Wohnungssuche oder bei Steuerberatungen. Zusätzlich führen diese Interviewten ins Feld, dass sie den Eindruck haben, dass BaslerInnen keine Ressourcen für neue Freunde haben“. (ebd.: 47)

Aus den gewonnenen Erkenntnissen leiten Wiener und Grossmann eine Anzahl von Handlungsempfehlungen für die Kantone beider Basel, internationale Unternehmen, gesellschaftliche Institutionen sowie die Expatriates selbst ab. (ebd.: 55ff.)

Daniel Wiener und Marco Grossmann sind in ihrer Studie den Spuren von Expatriates im Raum Basel nachgegangen. Einige ihrer Ergebnisse habe ich in meiner Untersuchung über Expatriates in Zug verwendet, auch wenn die thematische Ausrichtung ihrer Arbeit eine andere ist. Zwei weitere Studien haben mir wichtige Anregungen in meiner Auseinandersetzung mit Expatriates gegeben.

Die Studie „Immigration 2030“ (vgl. Stutz et al. 2010) - im Auftrag der Zürcher Kantonalbank - legt für den Wirtschaftsraum Zürich eine Ist-Analyse der ,neuen Zuwanderung“ sowie Szenarien für die weitere Entwicklung der Zürcher Wirtschaft und Gesellschaft vor. Fragen der freiwilligen, zivilgesellschaftlichen Beteiligungsmöglichkeiten von Expatriates kommen hingegen kaum zur Sprache. In einem qualitativen Teilprojekt zur Studie der Zürcher Kantonalbank wird auf Basis einer Gruppendiskussion $u$. a. das soziale Umfeld der neuen Zuwanderinnen und Zuwanderer untersucht. (Baghdadi 2010: 34)

Ebenfalls von Interesse ist meiner Meinung nach die Publikation von Daniel Müller-Jentsch und Avenir Suisse (2008) mit dem Titel „Die neue Zuwanderung. Die Schweiz zwischen BrainGain und Überfremdungsangst". Dieser Sammelband gibt anhand von Portraits und Interviews mit Expatriates sowie einigen journalistischen Milieustudien Einblicke in die Expatriate Lebenswelt in Zürich. (vgl. Von Arx 2008) Eine systematische Darstellung der Ansprüche, Ressourcen und Beteiligungsmöglichkeiten der Expatriates ist aber auch in diesem Werk nicht zu finden.

Anhand der dargestellten Literatur kann zusammengefasst werden, dass das freiwillige Engagement in der Schweiz (auch bei einem leichten Rückgang der formell freiwillig Tätigen) weit verbreitet ist und eine solide Basis für den gesellschaftlichen Zusammenhalt (vor allem auf der 
Gemeindeebene) bildet. Hierbei sind es vor allem Vereine und andere Freiwilligenorganisationen, in denen Engagement erlebt wird; sie bilden gewissermassen die Grundpfeiler des freiwilligen Engagements. Inwieweit diese Vereinskultur für das freiwillige, zivilgesellschaftliche Engagement von Expatriates in der Schweiz Beteiligungsmöglichkeiten bietet resp. welche (anderen resp. erweiterten) Ansprüche Expatriates überhaupt an zivilgesellschaftliche Beteiligungsformen stellen, ist bisher noch nicht systematisch erforscht. Mit meiner Studie möchte ich einen Beitrag zur Schliessung dieser Forschungslücke im Bereich der qualitativen Forschung zum freiwilligen Engagement von Expatriates in der Schweiz leisten.

Open Access Dieses Kapitel wird unter der Creative Commons Namensnennung 4.0 International Lizenz (http://creativecommons.org/licenses/by/4.0/deed.de) veröffentlicht, welche die Nutzung, Vervielfältigung, Bearbeitung, Verbreitung und Wiedergabe in jeglichem Medium und Format erlaubt, sofern Sie den/die ursprünglichen Autor(en) und die Quelle ordnungsgemäß nennen, einen Link zur Creative Commons Lizenz beifügen und angeben, ob Änderungen vorgenommen wurden.

Die in diesem Kapitel enthaltenen Bilder und sonstiges Drittmaterial unterliegen ebenfalls der genannten Creative Commons Lizenz, sofern sich aus der Abbildungslegende nichts anderes ergibt. Sofern das betreffende Material nicht unter der genannten Creative Commons Lizenz steht und die betreffende Handlung nicht nach gesetzlichen Vorschriften erlaubt ist, ist für die oben aufgeführten Weiterverwendungen des Materials die Einwilligung des jeweiligen Rechteinhabers einzuholen.

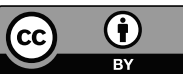

